\title{
Perturbative invariants of 3-manifolds with the first Betti number 1
}

\author{
TOMOTADA OHTSUKI
}

\begin{abstract}
It is known that perturbative invariants of rational homology 3-spheres can be constructed by using arithmetic perturbative expansion of quantum invariants of them. However, we could not make arithmetic perturbative expansion of quantum invariants for 3-manifolds with positive Betti numbers by the same method.

In this paper, we explain how to make arithmetic perturbative expansion of quantum $S O$ (3) invariants of 3-manifolds with the first Betti number 1. Further, motivated by this expansion, we construct perturbative invariants of such 3-manifolds. We show some properties of the perturbative invariants, which imply that their coefficients are independent invariants.
\end{abstract}

\section{$57 \mathrm{M} 27$}

In the late 1980s, Witten [44] proposed topological invariants of a closed 3-manifold $M$ for a simple compact Lie group $G$, what we call quantum $G$ invariant, which is formally presented by a path integral whose Lagrangian is the Chern-Simons functional of $G$ connections on $M$. There are two approaches to obtain mathematically rigorous information from a path integral: the operator formalism and the perturbative expansion. Motivated by the operator formalism of the Chern-Simons path integral, Reshetikhin and Turaev [34] gave the first rigorous mathematical construction of quantum invariants, as linear sums of quantum invariants of framed links. After that, rigorous constructions of quantum invariants were obtained by various approaches; in particular, Kirby and Melvin [14] constructed the quantum $S O(3)$ invariant, which we denote by $\tau_{r}^{S O(3)}(M)$; it is defined to be a linear sum of the quantum $\mathfrak{s l}_{2}$ invariant (the colored Jones polynomial) of framed links at an $r$ th roots of unity. On the other hand, the perturbative expansion of the Chern-Simons path integral suggests that we can construct perturbative invariants which describe asymptotic behavior of quantum invariants at $r \rightarrow \infty$; in fact, it is known (see, for example, the author's book [28]) that we can construct perturbative invariants of rational homology 3-spheres based on arithmetic perturbative expansion of quantum invariants of them.

We review the construction of the perturbative $S O(3)$ invariant of a rational homology $3-$ sphere $M$, as follows. Let $p$ be an odd prime, and put $\zeta=\exp (2 \pi \sqrt{-1} / p)$. Since 
it is known (by Murakami [23]) that $\tau_{p}^{S O(3)}(M) \in \mathbb{Z}[\zeta]$, we can make an expansion,

$$
\tau_{p}^{S O(3)}(M)=a_{p, 0}+a_{p, 1}(\zeta-1)+a_{p, 2}(\zeta-1)^{2}+\cdots+a_{p, N}(\zeta-1)^{N},
$$

with some integers $a_{p, n}$. Though this expansion is not unique, $\left(a_{p, n} \bmod p\right) \in \mathbb{Z} / p \mathbb{Z}$ is uniquely determined by the value of $\tau_{p}^{S O(3)}(M)$, since $\mathbb{Z}[\zeta]$ is isomorphic to $\mathbb{Z}[q] / T(q)$ where

$$
T(q)=\frac{q^{p}-1}{q-1}=\left(\begin{array}{l}
p \\
1
\end{array}\right)+\left(\begin{array}{l}
p \\
2
\end{array}\right)(q-1)+\left(\begin{array}{l}
p \\
3
\end{array}\right)(q-1)^{2}+\cdots+\left(\begin{array}{l}
p \\
p
\end{array}\right)(q-1)^{p-1} .
$$

The author showed in [26] that there exist $\lambda_{n}(M) \in \mathbb{Z}\left[\frac{1}{2}, \frac{1}{3}, \ldots, \frac{1}{m}\right]$, where $m=$ $\max \left\{2 n+1,\left|H_{1}(M ; \mathbb{Z})\right|\right\}$ and $\left|H_{1}(M ; \mathbb{Z})\right|$ denotes the order of $H_{1}(M ; \mathbb{Z})$, such that

$$
a_{p, n} \underset{(p)}{\equiv}\left(\frac{\left|H_{1}(M ; \mathbb{Z})\right|}{p}\right) \lambda_{n}(M)
$$

for any odd prime $p \geq \max \left\{2 n+3,\left|H_{1}(M ; \mathbb{Z})\right|\right\}$, and defined the perturbative $S O(3)$ invariant of $M$ by

$$
\tau(M)=\lambda_{0}(M)+\lambda_{1}(M)(q-1)+\lambda_{2}(M)(q-1)^{2}+\cdots \in \mathbb{Q} \llbracket q-1 \rrbracket .
$$

We call this the arithmetic perturbative expansion of $\tau_{p}^{S O(3)}(M)$. In particular, by results of Murakami [23], we can see that the first two coefficients are presented by "(semi-)classical" invariants,

$$
\lambda_{0}(M)=\frac{1}{\left|H_{1}(M ; \mathbb{Z})\right|}, \quad \lambda_{1}(M)=\frac{6 \lambda(M)}{\left|H_{1}(M ; \mathbb{Z})\right|},
$$

where $\lambda(M)$ denotes the Casson-Walker invariant [43] of $M$. Further, when $M$ is obtained from $S^{3}$ by $f$ surgery along a knot $K, \tau(M)$ is presented by Gaussian integral of the following form (see Rozansky [38; 40], Bar-Natan, Garoufalidis, Rozansky and Thurston $[1 ; 2 ; 3]$ and the author's book [28]),

$$
\left.\tau(M)=(\text { constant }) \cdot \int_{n \in \mathbb{R}} q^{f\left(n^{2}-1\right) / 4}[n]^{2} \text { (perturbative expansion of } J_{n}(K ; q)\right) d n,
$$

where $[n]=\left(q^{n / 2}-q^{-n / 2}\right) /\left(q^{1 / 2}-q^{-1 / 2}\right)$, and $J_{n}(K ; q)$ denotes the colored Jones polynomial of $K$, whose perturbative expansion is a power series in $q-1$ with coefficients of polynomials in $q$ and $q^{n}$. Note that, when $f=0$, this integral diverges.

In this paper, motivated by the arithmetic perturbative expansion of $\tau_{p}^{S O(3)}(M)$, we construct a perturbative invariant of a 3-manifold $M$ with the first Betti number 1,

$$
\tau(M ; c)=\lambda_{0}(M ; c)+\lambda_{1}(M ; c)(q-1)+\lambda_{2}(M ; c)(q-1)^{2}+\cdots \in \mathbb{C} \llbracket q-1 \rrbracket,
$$


where $c$ is 0 or a zero of the Alexander polynomial $\Delta(t)$ of $M$, and prove that it is a topological invariant of $M$ (Theorem 1.1). When $M$ is obtained from $S^{3}$ by 0 surgery along a knot $K$, instead of the $f=0$ case of the above Gaussian integral, we consider the following integral,

$$
\text { (constant) } \left.\cdot \int_{t \in\{|z|=1\} \subset \mathbb{C}} \text { (loop expansion of }\left.[n]^{2} J_{n}(K ; q)\right|_{q^{n} \rightarrow t}\right) \frac{d t}{t},
$$

where the loop expansion of $J_{n}(K ; q)$ is a power series in $q-1$ with coefficients of rational functions of $q^{n}$ whose denominators are powers of the Alexander polynomial of $K$, and our construction of the perturbative invariant is obtained as the residue of this integral,

$$
\tau(M ; c)=(\text { constant }) \cdot \operatorname{Res} \frac{1}{t=c}\left(\text { loop expansion of }\left.[n]^{2} J_{n}(K ; q)\right|_{q^{n} \rightarrow t}\right) .
$$

The 0 th coefficient $\lambda_{0}(M ; c)$ is presented by the Alexander polynomial of $M$ (Proposition 5.3), noting that the Alexander polynomial can be regarded as the $\mathbb{Z}$ equivariant version of the order of the first homology group. Further, when $M$ is obtained from $S^{3}$ by 0 surgery along a knot $K, \lambda_{1}(M ; c)$ is presented by the 2-loop polynomial of $K$, which can be regarded as " $\mathbb{Z}$ equivariant Casson invariant" of the infinite cyclic cover of the complement of $K$ (see the author's paper [29]). $\lambda_{1}(M ; c)$ can be regarded as another extension of the Casson-Walker invariant than the Casson-Walker-Lescop invariant (see Lescop [20; 21]), for 3-manifolds with the first Betti number 1. The coefficients of the arithmetic expansion of $\tau_{p}^{S O(3)}(M)$ are presented by linear sums of 1 and $\left(\left(1+c_{i}\right) /\left(1-c_{i}\right)\right)^{p}$, where $c_{1}^{ \pm 1}, \ldots, c_{n}^{ \pm 1}$ are the zeros of the Alexander polynomial. As we explain in Section 2, the space of such series can be described by the "Tamagawa map",

$$
\left(\sum_{i=1}^{n}\left(\frac{1+c_{i}}{1-c_{i}}\right)^{a p+b}\right)_{p: \text { primes }} \longmapsto \sum_{i=1}^{n}\left(\frac{1+c_{i}}{1-c_{i}}\right)^{a} \otimes\left(\frac{1+c_{i}}{1-c_{i}}\right)^{b} \in \mathbb{C} \otimes \mathbb{Q} \mathbb{C}
$$

Through this map, we can regard

$$
\sum_{c=0, c_{1}, \ldots, c_{n}} \frac{1+c}{1-c} \otimes \frac{1-c}{1+c} \tau(M ; c) \in\left(\mathbb{C} \otimes_{\mathbb{Q}} \mathbb{C}\right) \llbracket q-1 \rrbracket
$$

as an arithmetic perturbative expansion of $\tau_{p}^{S O(3)}(M)$.

An idea of constructing our perturbative invariants is to consider equivariant invariants (see the author's papers $[29 ; 32 ; 31]$ ). As mentioned above, the Alexander polynomial and the 2-loop polynomial can be regarded as equivariant invariants. More generally, the loop expansion of the Kontsevich invariant of a knot can be regarded as the " $\mathbb{Z}$ 
equivariant LMO invariant" of the infinite cyclic cover of the knot complement. As its $\mathfrak{s l}_{2}$ reduction, the loop expansion of the colored Jones polynomial of a knot can be regarded as the " $\mathbb{Z}$ equivariant perturbative $S O(3)$ invariant" of the infinite cyclic cover of the knot complement. In this sense, our perturbative invariants of 3-manifolds with the first Betti number 1 can be regarded as " $\mathbb{Z}$ equivariant perturbative invariants" of the infinite cyclic cover of such 3-manifolds. By considering equivariant invariants of covering spaces, we can construct perturbative invariants of such 3-manifolds, though we could not define perturbative invariants of such 3-manifolds by the method for homology 3-spheres.

It is known that, though the LMO invariant (see Le, Murakami and Ohtsuki [19]) is powerful enough to be expected to classify integral homology 3-spheres, it is weak for 3-manifolds with positive first Betti numbers; in fact, when $b_{1}(M)>0$, the value of the LMO invariant of $M$ can be determined from "classical" invariants such as the cohomology ring, the Alexander polynomial and the Casson-Walker-Lescop invariant of $M$ (see Garoufalidis-Habegger [6], Habegger [10], Habegger-Beliakova [11] and Lieberum [22]). On the other hand, Proposition 5.2 suggests that our perturbative invariant is as fine as the perturbative $S O(3)$ invariant for homology 3 -spheres in the sense that the perturbative $S O(3)$ invariant of a homology 3-sphere $N$ can be determined from the values of our perturbative invariants of $M \# N$ and $M$ for a 3-manifold $M$ with the first Betti number 1. Moreover, Proposition 5.4 implies that the coefficients of our perturbative invariants are independent invariants. Since the LMO invariant dominates all perturbative invariants for homology 3-spheres (see the author's book [28]), it is expected that there exists a refinement of the LMO invariant; this will be discussed in [25]. Out perturbative invariants would be the $\mathfrak{s l}_{2}$ reduction of such a refinement of the LMO invariant.

It is still an open problem to interpret our perturbative invariants from the ChernSimons path integral. It might be necessary to consider a $\mathbb{Z}$ equivariant version of the Chern-Simons theory. It might be related to the fact that the space of irreducible $S U(2)$ representations of the fundamental group of a knot complement branches from the space of reducible representations at zeros of the Alexander polynomial of the knot.

The paper is organized as follows. In Section 1, we define perturbative invariants for 3-manifolds with the first Betti number 1, and show their concrete values for some examples. In Section 2, we explain how we can describe the arithmetic perturbative expansion of $\tau_{p}^{S O(3)}(M)$ in terms of the perturbative invariants. In Section 3, we review the loop expansions of the Kontsevich invariant and the colored Jones polynomials, which are used in the proof of the main theorem. In Section 4, we prove the main theorem, which states the topological invariance of the perturbative invariants. In Section 5, we show some properties of the perturbative invariants. In Section 6, we 
calculate arithmetic limits of some rational functions of roots of unity, which we use when we calculate arithmetic expansion of quantum invariants.

The author would like to thank Akio Tamagawa for the description of the space of certain arithmetic series, and Lev Rozansky for discussion on the loop expansion of the colored Jones polynomial. He is also grateful to Kazuo Habiro, Andrew Kricker, Tomoyoshi Yoshida, Christine Lescop, Jørgen Andersen, Gregor Masbaum, Thang Le for valuable comments and suggestions. He would also like to thank the referee for many helpful comments.

\section{Notation}

We denote by $\Delta(t)$ the Alexander polynomial of a knot $K$ or a 3-manifold $M$ with $b_{1}(M)=1$; they are equal when $M$ is obtained from $S^{3}$ by 0 surgery along $K$. We normalize $\Delta(t)$ in such a way that $\Delta(t)=\Delta\left(t^{-1}\right)$ and $\Delta(1)$ is equal to the order of the torsion subgroup of $H_{1}(M ; \mathbb{Z})$. Further, we normalize the colored Jones polynomial $J_{n}(K ; q)$ in such a way that $J_{n}$ (the trivial knot;q) $=1$. We note that $[n] J_{n}(K ; q)$ is equal to the quantum $\left(\mathfrak{s l}_{2}, V_{n}\right)$ invariant for any knot $K$ with 0 framing, which is the invariant defined from the irreducible $n$-dimensional representation $V_{n}$ of the quantum group of $\mathfrak{s l}_{2}$ (see, for example, the author's book [28]).

For a prime number $p$, we denote by $\mathbb{Z}_{(p)}$ the subring of $\mathbb{Q}$ consisting of rational numbers whose denominators are not divisible by $p$. For $x, y \in \mathbb{Z}_{(p)}$, we write $x \equiv \underset{(p)}{\equiv} y$ if $x-y$ is divisible by $p$ in $\mathbb{Z}_{(p)}$. Let $\mathcal{F}$ be an extension of $\mathbb{Q}$, let $\mathcal{O}$ be the integer ring of $\mathcal{F}$, and let $\mathfrak{p}$ be a prime ideal of $\mathcal{O}$. We denote by $\mathcal{O}_{(\mathfrak{p})}$ the subring of $\mathcal{F}$ consisting of elements of the form $a / b$ for $a \in \mathcal{O}$ and $b \in \mathcal{O}-\mathfrak{p}$. For $x, y \in \mathcal{O}_{(\mathfrak{p})}$, we write $x \underset{(\mathfrak{p})}{\equiv} y$ if $x-y \in \mathfrak{p} \mathcal{O}_{(\mathfrak{p})}$.

For Jacobi diagrams $D_{1}$ and $D_{2}$, we write $D_{1} \underset{\left(\mathfrak{s l}_{2}\right)}{\equiv} D_{2}$ if their images by the $\mathfrak{s l}_{2}$ weight system are equal.

\section{Definition of the perturbative invariants}

In this section, we define perturbative invariants for 3-manifolds with the first Betti number 1 , motivated by the arithmetic perturbative expansion of the quantum $S O(3)$ invariant. We show concrete values of the perturbative invariants for some examples and for the case where the Alexander polynomial has small degree.

Before explaining the general case, for simplicity, we explain the definition when $M$ is obtained from $S^{3}$ by surgery along a knot $K$ with 0 framing. Let $p$ be an odd prime, 
and put $\zeta=\exp (2 \pi \sqrt{-1} / p)$. Then, the quantum $S O(3)$ invariant $\tau_{p}^{S O(3)}(M)$ of $M$ is presented by

$$
\tau_{p}^{S O(3)}(M)=\frac{1}{c_{0}} \sum_{\substack{1 \leq n<p \\ n \text { is odd }}}[n]_{\zeta}^{2} J_{n}(K ; \zeta)
$$

where $J_{n}(K ; q)$ denotes the colored Jones polynomial of $K$,

$$
\text { and } \begin{aligned}
{[n]_{\zeta} } & =\left(\zeta^{n / 2}-\zeta^{-n / 2}\right) /\left(\zeta^{1 / 2}-\zeta^{-1 / 2}\right) \\
c_{0} & =\sum_{\substack{1 \leq n<p \\
n \text { is odd }}}[n]_{\zeta}^{2}=\frac{1}{2} \sum_{n \in \mathbb{Z} / p \mathbb{Z}} \frac{\zeta^{n}+\zeta^{-n}-2}{\zeta+\zeta^{-1}-2}=\frac{-p}{\zeta+\zeta^{-1}-2} .
\end{aligned}
$$

Further, as shown by Rozansky [41] (see also Remark 3.2), the loop expansion of the colored Jones polynomial is presented by

$$
J_{n}(K ; q)=\sum_{\ell=0}^{\infty} \frac{P_{\ell}\left(q^{n}\right)}{\Delta\left(q^{n}\right)^{2 \ell+1}}(q-1)^{\ell},
$$

where $P_{\ell}(t) \in \mathbb{Z}\left[t^{ \pm 1}\right]$ and, in particular, $P_{0}(t)=1$, and $\Delta(t)$ is the Alexander polynomial of $K$, which is equal to the Alexander polynomial of $M$. Hence, for each $n$,

$$
J_{n}(K ; \zeta)=\sum_{\ell=0}^{N} \frac{P_{\ell}\left(\zeta^{n}\right)}{\Delta\left(\zeta^{n}\right)^{2 \ell+1}}(\zeta-1)^{\ell}+O\left((\zeta-1)^{N+1} ; \mathbb{Z}_{(p)}[\zeta]\right) .
$$

By substituting this to the formula of $\tau_{p}^{S O(3)}(M)$,

$$
\begin{aligned}
\tau_{p}^{S O(3)}(M) & =\frac{1}{c_{0}} \sum_{\substack{1 \leq n<p \\
n \text { is odd }}} \frac{\zeta^{n}+\zeta^{-n}-2}{\zeta+\zeta^{-1}-2} \sum_{\ell=0}^{N} \frac{P_{\ell}\left(\zeta^{n}\right)}{\Delta\left(\zeta^{n}\right)^{2 \ell+1}}(\zeta-1)^{\ell}+O\left((\zeta-1)^{N+1}\right) \\
& =-\frac{1}{2} \sum_{\ell=0}^{N}\left(\frac{1}{p} \sum_{n \in \mathbb{Z} / p \mathbb{Z}} \frac{\left(\zeta^{n}+\zeta^{-n}-2\right) P_{\ell}\left(\zeta^{n}\right)}{\Delta\left(\zeta^{n}\right)^{2 \ell+1}}\right)(\zeta-1)^{\ell}+O\left((\zeta-1)^{N+1}\right) .
\end{aligned}
$$

Further, by Proposition $6.1, \tau_{p}^{S O(3)}(M) \in \mathbb{Z}_{(p)}[\zeta]$ and

$$
\begin{array}{r}
\tau_{p}^{S O(3)}(M)=-\frac{1}{2} \sum_{\ell=0}^{N}\left(\sum_{c=0, c_{1}, \ldots, c_{n}}\left(\frac{1+c}{1-c}\right)^{p} \operatorname{Res}_{t=c} \frac{\left(1-t^{-1}\right)^{2} P_{\ell}(t)}{\Delta(t)^{2 \ell+1}}\right)(\zeta-1)^{\ell} \\
+O\left((\zeta-1)^{\min \{N+1, p-1\}}\right)
\end{array}
$$


for all but finitely many primes $p$, where $c_{1}, c_{1}^{-1}, \ldots, c_{n}, c_{n}^{-1}$ are the zeros of $\Delta(t)$. Motivated by this formula, we define the perturbative invariant of $M$ at $c$ by

$$
\tau(M ; c)=-\frac{1}{2} \cdot \frac{1+c}{1-c} \sum_{\ell=0}^{\infty}\left(\operatorname{Res}_{t=c} \frac{\left(1-t^{-1}\right)^{2} P_{\ell}(t)}{\Delta(t)^{2 \ell+1}}\right)(q-1)^{\ell} \in \mathbb{C} \llbracket q-1 \rrbracket,
$$

where $c$ is 0 or a zero of the Alexander polynomial $\Delta(t)$ of $M$.

In general, we define the perturbative invariant, as follows. Let $M$ be a 3-manifold with the first Betti number 1 . Then, $M$ can be obtained from a rational homology 3-sphere $N$ by surgery along a null-homologous knot $K$ with 0 framing in $N$. By Proposition 3.1,

$$
W_{\mathfrak{s} \mathfrak{L}_{2}, V_{n}}\left(Z^{\mathrm{LMO}}(N, K)\right)=[n] \sum_{\ell=0}^{\infty} \frac{P_{\ell}\left(q^{n}\right)}{\Delta\left(q^{n}\right)^{2 \ell+1}}(q-1)^{\ell}
$$

for some $P_{\ell}(t) \in \mathbb{Q}\left[t^{ \pm 1}\right]$, where $\Delta(t)$ is the Alexander polynomial of $K$, which is equal to the Alexander polynomial of $M$; in particular, $P_{0}(t)(=\Delta(1))$ is equal to the order of the torsion part of $H_{1}(M ; \mathbb{Z})$. By using these $P_{\ell}(t)$, we define the perturbative invariant $\tau(M ; c)$ at $c$ by (3), where $c$ is 0 or a zero of the Alexander polynomial $\Delta(t)$ of $M$. By Remark 3.2, this definition fits the previous definition when $N=S^{3}$.

Theorem $1.1 \tau(M ; c)$ does not depend on the choice of $N$ and $K$, that is, $\tau(M ; c)$ is a topological invariant of a 3-manifold $M$ with $b_{1}(M)=1$.

We show the proof of the theorem in Section 4.

By definition, $\tau(M ; 0) \in \mathbb{Q} \llbracket q-1 \rrbracket$. By Proposition 1.6, if the irreducible factors of $\Delta(t)$ are of degree $\leq 1$, then $\tau(M ; c) \in \mathbb{Q} \llbracket q-1 \rrbracket$. By Proposition 5.1, $\tau(M ; c)=\tau\left(M ; c^{-1}\right)$ for a zero $c$ of $\Delta(t)$. We denote by $\lambda_{\ell}(M ; c)$ the $\ell$ th coefficient of $\tau(M ; c)$, that is, $\tau(M ; c)=\sum_{\ell=0}^{\infty} \lambda_{\ell}(M ; c)(q-1)^{\ell}$ and

$$
\lambda_{\ell}(M ; c)=-\frac{1}{2} \cdot \frac{1+c}{1-c} \operatorname{Res} \frac{\left(1-t^{-1}\right)^{2} P_{\ell}(t)}{\Delta(t)^{2 \ell+1}} \in \mathbb{C} .
$$

Note that $\lambda_{\ell}(M ; 0) \in \mathbb{Q}$, and, if the irreducible factors of $\Delta(t)$ are of degree $\leq 1$, then $\lambda_{\ell}(M ; c) \in \mathbb{Q}$.

Example 1.2 By definition, $\tau\left(S^{1} \times S^{2} ; 0\right)=1$, since $S^{1} \times S^{2}$ is obtained from $S^{3}$ by 0 surgery along the trivial knot. Hence, by Proposition 5.2, for a rational homology 3-sphere $N, \tau\left(\left(S^{1} \times S^{2}\right) \# N ; 0\right)$ is equal to the product of the perturbative $S O(3)$ invariant (see the author's paper [26]) of $N$ and the order of $H_{1}(N ; \mathbb{Z})$. 
Example 1.3 For the 3-manifold obtained from $S^{3}$ by 0 surgery along the $(a, b)$ torus knot,

$$
\tau(M ; 0)= \begin{cases}-\frac{1}{2} q & \text { if }(a, b)=(2,3), \quad \tau(M ; c)=\frac{1-c^{-2}}{2 \Delta^{\prime}(c)} q^{\left(a-a^{-1}\right)\left(b-b^{-1}\right) / 4}, \\ 0 & \text { otherwise, }\end{cases}
$$

where $c$ is a zero of

$$
\Delta(t)=\frac{\left(t^{a b / 2}-t^{-a b / 2}\right)\left(t^{1 / 2}-t^{-1 / 2}\right)}{\left(t^{a / 2}-t^{-a / 2}\right)\left(t^{b / 2}-t^{-b / 2}\right)}
$$

and we regard the right-hand sides of the formulas as in $\mathbb{Q} \llbracket q-1 \rrbracket$ by expanding them into power series in $q-1$.

Proof Rozansky [39] showed that the loop expansion of the colored Jones polynomial is presented by

$$
J_{n}\left(K ; q^{n}\right)=\frac{q^{(a b-a / b-b / a) / 4}}{t^{1 / 2}-t^{-1 / 2}} \sum_{m=0}^{\infty} \frac{1}{m !}\left(\frac{\log q}{a b}\right)^{m}\left(t \frac{d}{d t}\right)^{2 m} \frac{t^{1 / 2}-t^{-1 / 2}}{\Delta(t)},
$$

where the equality holds by putting $t=q^{n}$ after we calculate the right-hand side. Hence, by definition,

$$
\begin{aligned}
& \tau(M ; c)=-\frac{q^{(a b-a / b-b / a) / 4}}{2} \cdot \frac{1+c}{1-c} \\
& \times \sum_{m=0}^{\infty} \frac{1}{m !}\left(\frac{\log q}{a b}\right)^{m} \operatorname{Res}_{t=c}^{m} \frac{t^{1 / 2}-t^{-1 / 2}}{t}\left(t \frac{d}{d t}\right)^{2 m} \frac{t^{1 / 2}-t^{-1 / 2}}{\Delta(t)} .
\end{aligned}
$$

The residue of this formula is calculated recursively by using

$$
\operatorname{Res}_{t=c}\left(t^{1 / 2} \mp t^{-1 / 2}\right) \frac{d}{d t}(t \varphi(t))=-\frac{1}{2} \operatorname{Res}_{t=c}\left(t^{1 / 2} \pm t^{-1 / 2}\right) \varphi(t),
$$

where we obtain this formula from

$$
\frac{d}{d t}\left(\left(t^{1 / 2} \mp t^{-1 / 2}\right)(t \varphi(t))\right)=\frac{1}{2}\left(t^{1 / 2} \pm t^{-1 / 2}\right) \varphi(t)+\left(t^{1 / 2} \mp t^{-1 / 2}\right) \frac{d}{d t}(t \varphi(t)),
$$

since the residue of the differential of some function equals 0 . It follows that

$$
\begin{aligned}
\tau(M ; c) & =-\frac{q^{(a b-a / b-b / a) / 4}}{2} \cdot \frac{1+c}{1-c} \sum_{m=0}^{\infty} \frac{1}{m !}\left(\frac{\log q}{a b}\right)^{m} \times \frac{1}{4^{m}} \operatorname{Res} \frac{t+t^{-1}-2}{t \Delta(t)} \\
& =-\frac{q^{\left(a-a^{-1}\right)\left(b-b^{-1}\right) / 4}}{2} \cdot \frac{1+c}{1-c} \operatorname{Res} \frac{t+t^{-1}-2}{t \Delta(t)} .
\end{aligned}
$$


When $c=0$, we calculate $\tau(M ; 0)$, as follows. If $(a, b)=(2,3)$, then $\Delta(t)=$ $t-1+t^{-1}$, and we obtain the required formula by concrete computation. Otherwise, $\operatorname{deg} \Delta(t)>1$. Hence, putting

$$
\Delta(t)=\left(t^{n}+t^{-n}\right)+\cdots,
$$

the function of the residue has the form

$$
\frac{t^{n}-2 t^{n-1}+t^{n-2}}{t^{2 n}+\cdots+1}
$$

and it has no pole at $t=0$. Therefore, its residue equals 0 , and we obtain the required formula.

When $c$ is a zero of $\Delta(t)$, the residue is calculated as

$$
\operatorname{Res}_{t=c} \frac{t+t^{-1}-2}{t \Delta(t)}=\lim _{t \rightarrow c} \frac{(t-c)\left(t+t^{-1}-2\right)}{t \Delta(t)}=\lim _{t \rightarrow c} \frac{t+t^{-1}-2}{t \Delta^{\prime}(t)}=\frac{(c-1)^{2}}{c^{2} \Delta^{\prime}(c)} .
$$

Hence, by using this formula, we obtain the required formula.

Example 1.4 Rozansky [39] calculated "approximation" of loop polynomials of some knots. If his "approximate" formulas would be the exact ones, we can calculate our invariants by using them for 3-manifolds obtained from $S^{3}$ by 0 surgery along those knots. For example, for the 3-manifold $M$ obtained from $S^{3}$ by 0 surgery along the figure-eight knot,

$$
\begin{gathered}
\tau(M ; 0)=\frac{1}{2}+0(q-1)+0(q-1)^{2}+\cdots, \\
\tau\left(M ; \frac{3+\sqrt{5}}{2}\right)=-\frac{1}{2}+0(q-1)-\frac{1}{125}(q-1)^{2}+\cdots .
\end{gathered}
$$

Further, for the 3-manifold $M$ obtained from $S^{3}$ by 0 surgery along the $5_{2}$ knot,

$$
\begin{gathered}
\tau(M ; 0)=-\frac{1}{4}+\frac{5}{16}(q-1)-\frac{23}{64}(q-1)^{2}+\cdots, \\
\tau\left(M ; \frac{3+\sqrt{-7}}{4}\right)=\frac{1}{4}-\frac{31}{112}(q-1)+\frac{915}{3126}(q-1)^{2}+\cdots .
\end{gathered}
$$

The 0 th and 1 st coefficients of the right-hand sides are correct, since we can calculate the 2-loop polynomial exactly for arbitrarily given knot; see Kricker [18] and the author's papers [30;33]. 


\subsection{The case where $\Delta(t)$ is of degree 0}

In this case, $\Delta(t)=b$, where $b$ is the order of the torsion subgroup of $H_{1}(M ; \mathbb{Z})$. We can calculate the perturbative invariant from the loop polynomial by

$$
\begin{aligned}
\lambda_{\ell}(M ; 0) & =-\frac{1}{2} \operatorname{Res} \frac{\left(t+t^{-1}-2\right) P_{\ell}(t)}{b^{2 \ell+1} t} \\
& =-\frac{1}{2 b^{2 \ell+1}}\left(\text { constant term of }\left(t+t^{-1}-2\right) P_{\ell}(t)\right) .
\end{aligned}
$$

Remark 1.5 When $H_{1}(M ; \mathbb{Z}) \cong \mathbb{Z}$ and the Alexander polynomial $\Delta(t)$ of $M$ equals 1, independently of the author, Kazuo Habiro and Thang Le constructed an invariant, presented by "Habiro expansion", which is an expansion of the form

$$
\sum_{n=0}^{\infty} a_{n}(q-1)\left(q^{2}-1\right) \ldots\left(q^{n}-1\right) .
$$

It is an equivalent invariant to $\tau(M ; 0)$ for such 3 -manifolds in the sense that their invariant is uniquely determined by $\tau(M ; 0)$. Further, they showed that the value of each $\tau_{p}^{S O(3)}(M)$ is obtained from their invariant by substituting a root of unity to $q$. It follows that $\tau(M ; 0)$ is universal among quantum invariants $\tau_{p}^{S O(3)}(M)$ for such 3-manifolds.

\subsection{The case where the irreducible factors of $\Delta(t)$ are of degree 1}

In this case, we show Propositions 1.6 and 1.7, in this subsection.

Proposition 1.6 If the irreducible factors of $\Delta(t)$ are of degree $1, \tau(M ; c) \in \mathbb{Q} \llbracket q-1 \rrbracket$.

Proof It is sufficient to show that $\lambda_{\ell}(M ; c) \in \mathbb{Q}$ for a zero $c$ of $\Delta(t)$, where $c$ is a zero of a factor $1-a\left(t+t^{-1}-2\right)$ of $\Delta(t)$. By definition,

$$
\lambda_{\ell}(M ; c)=-\frac{1}{2 b_{0}^{2 \ell+1}} \cdot \frac{1+c}{1-c} \operatorname{Res} \frac{\left(t+t^{-1}-2\right) P_{\ell}(t)}{t \Delta(t)^{2 \ell+1}} .
$$

It can be presented by a linear sum of

$$
\frac{1+c}{1-c} \operatorname{Res}_{t=c} \frac{\left(t+t^{-1}-2\right)^{m}}{t\left(1-a\left(t+t^{-1}-2\right)\right)^{m+1}} .
$$

Further, by Lemma 6.5, this is equal to

$$
(-1)^{m} 2\left(\begin{array}{c}
2 m-1 \\
m
\end{array}\right)\left(\frac{1-c}{1+c}\right)^{2 m}=\frac{(-1)^{m} 2}{(4 a+1)^{m}}\left(\begin{array}{c}
2 m-1 \\
m
\end{array}\right) \in \mathbb{Q},
$$


since $4 a+1=(1+c)^{2} /(1-c)^{2}$. Hence, $\lambda_{\ell}(M ; c) \in \mathbb{Q}$.

Proposition 1.7 If $\Delta(t)$ is of degree 1, the following (1) and (2) holds, where we put $\Delta(t)=b_{0}-b_{1}\left(t+t^{-1}-2\right)$ with non-zero integers $b_{0}, b_{1}$, and $c$ is a zero of $\Delta(t)$.

(1) The 0 th coefficients of the perturbative invariants are presented by

$$
\lambda_{0}(M ; 0)=\frac{b_{0}}{2 b_{1}}, \quad \lambda_{0}(M ; c)=-\frac{b_{0}}{2 b_{1}} .
$$

(2) Putting the 2-loop polynomial by $P_{1}(t)=f(t) \Delta(t)^{3}+a_{2} \Delta(t)^{2}+a_{1} \Delta(t)+a_{0}$, the first coefficients of the perturbative invariants are presented by

$$
\begin{aligned}
& \lambda_{1}(M ; 0)=-\frac{1}{2}\left(\text { constant term of }\left(t+t^{-1}-2\right) f(t)\right)+\frac{a_{2}}{2 b_{1}}, \\
& \lambda_{1}(M ; c)=-\frac{a_{2}}{2 b_{1}}+\frac{a_{1}}{b_{0}\left(4 b_{1}+b_{0}\right)}+\frac{a_{0}\left(b_{1}+b_{0}\right)}{b_{0}^{2}\left(4 b_{1}+b_{0}\right)^{2}} .
\end{aligned}
$$

Proof We obtain (1) by Proposition 5.3.

We show (2), as follows. By definition, putting $z=t+t^{-1}-2$,

$$
\begin{aligned}
\lambda_{1}(M ; 0) & =-\frac{1}{2} \operatorname{Res} \frac{z P_{1}(t)}{t=0(t)^{3}} \\
& =-\frac{1}{2} \operatorname{Res} \frac{z}{t=0}\left(f(t)+\frac{a_{2}}{b_{0}-b_{1} z}+\frac{a_{1}}{\left(b_{0}-b_{1} z\right)^{2}}+\frac{a_{0}}{\left(b_{0}-b_{1} z\right)^{3}}\right) .
\end{aligned}
$$

Since

$$
\operatorname{Res}_{t=0} \frac{z}{t\left(b_{0}-b_{1} z\right)^{\ell}}= \begin{cases}-\frac{1}{b_{1}} & \text { if } \ell=1, \\ 0 & \text { if } \ell>1,\end{cases}
$$

we obtain the required formula of $\lambda_{1}(M ; 0)$. Similarly, we have that

$$
\lambda_{1}(M ; c)=-\frac{1}{2} \cdot \frac{1+c}{1-c} \operatorname{Res} \frac{z}{t=c}\left(f(t)+\frac{a_{2}}{b_{0}-b_{1} z}+\frac{a_{1}}{\left(b_{0}-b_{1} z\right)^{2}}+\frac{a_{0}}{\left(b_{0}-b_{1} z\right)^{3}}\right) .
$$

Since

$$
\frac{1+c}{1-c} \operatorname{Res}_{t=c} \frac{z}{t\left(b_{0}-b_{1} z\right)^{\ell}}= \begin{cases}\frac{1}{b_{1}} & \text { if } \ell=1, \\ -\frac{2}{b_{0}\left(4 b_{1}+b_{0}\right)} & \text { if } \ell=2, \\ -\frac{2\left(b_{1}+b_{0}\right)}{b_{0}^{2}\left(4 b_{1}+b_{0}\right)^{2}} & \text { if } \ell=3,\end{cases}
$$

we obtain the required formula of $\lambda_{1}(M ; c)$. 


\section{Arithmetic perturbative expansion of $\tau_{p}^{S o(3)}(M)$}

In this section, we explain how we can describe the arithmetic perturbative expansion of $\tau_{p}^{S O(3)}(M)$. We define $\tau(M ; \sigma)$, which describes the behavior of the series $\left(\hat{\lambda}_{p, \ell}(M)\right)$ for certain subsequences of $p$. Further, we define $\tau(M)$, which is obtained from the vector $(\tau(M ; \sigma))_{\sigma}$ by such a coordinate change that the resulting $\tau(M)$ does not depend on $\sigma$.

We briefly review some preliminaries of number theory used in this section; for details see, for example, Neukirch [24] and Ribenboim [35]. We denote by $\mathcal{F}$ the minimal splitting field of $\Delta(t)$, which is a Galois extension of $\mathbb{Q}$. We denote by $\mathcal{O}$ the integer ring of $\mathcal{F}$. It is known that, for any prime number $p$, the ideal $p \mathcal{O} \subset \mathcal{O}$ decomposes into the form,

$$
p \mathcal{O}=\left(\mathfrak{p}_{1} \ldots \mathfrak{p}_{g}\right)^{e}
$$

where the $\mathfrak{p}_{i}$ s are prime ideals in $\mathcal{O}$ such that $\mathfrak{p}_{i} \cap \mathbb{Z}=(p)$; they are related to each other by $\mathfrak{p}_{j}=\sigma\left(\mathfrak{p}_{i}\right)$ for some $\sigma \in \operatorname{Gal}(\mathcal{F} / \mathbb{Q})$. When $e=1, \mathfrak{p}_{i}$ is called unramified. Let $\mathfrak{p}$ denote a prime ideal in $\mathcal{O}$ such that $(p)=\mathfrak{p} \cap \mathbb{Z}$. It is known that $\mathfrak{p}$ is unramified for all but finitely many $\mathfrak{p}$. The decomposition group of $\mathfrak{p}$ is defined by

$$
D_{\mathfrak{p}}=\{\sigma \in \operatorname{Gal}(\mathcal{F} / \mathbb{Q}) \mid \sigma(\mathfrak{p})=\mathfrak{p}\} .
$$

Let $k_{\mathfrak{p}}$ denote the residue field $\mathcal{O} / \mathfrak{p}$, which is a Galois extension of the prime field $\mathbb{F}_{p}$. It is known that, when $\mathfrak{p}$ is unramified, the left map of the following line is an isomorphism,

$$
\operatorname{Gal}\left(k_{\mathfrak{p}} / \mathbb{F}_{p}\right) \stackrel{\cong}{\leftrightarrows} D_{\mathfrak{p}} \subset \operatorname{Gal}(\mathcal{F} / \mathbb{Q}) .
$$

Let $\operatorname{Fr}_{\mathfrak{p}}$ denote the Frobenius map $k_{\mathfrak{p}} \rightarrow k_{\mathfrak{p}}$ taking $x$ to $x^{p}$. It is known that $\operatorname{Gal}\left(k_{\mathfrak{p}} / \mathbb{F}_{p}\right)$ is generated by $\operatorname{Fr}_{\mathfrak{p}}$. When $\mathfrak{p}$ is unramified, by the above map, we associate the Frobenius automorphism $\operatorname{Fr}_{\mathfrak{p}}=\sigma \in \operatorname{Gal}(\mathcal{F} / \mathbb{Q})$ with $\mathfrak{p}$. Let $\mathcal{P}$ be the set of unramified prime ideals of $\mathcal{O}$. For each $\sigma \in \operatorname{Gal}(\mathcal{F} / \mathbb{Q})$, we put $\mathcal{P}(\sigma)$ to be the set of $\mathfrak{p} \in \mathcal{P}$ whose Frobenius automorphism is $\sigma$, that is,

$$
\mathcal{P}(\sigma)=\left\{\mathfrak{p} \in \mathcal{P} \mid \mathrm{Fr}_{\mathfrak{p}}=\sigma\right\} .
$$

When $\mathfrak{p} \in \mathcal{P}(\sigma), \tau(\mathfrak{p}) \in \mathcal{P}\left(\tau \sigma \tau^{-1}\right)$ for $\tau \in \operatorname{Gal}(\mathcal{F} / \mathbb{Q})$. It is known by the Chebotarev density theorem (see, for example, Neukirch [24, Theorem VII.13.4] and Ribenboim [35, Section 25.3]) that $\mathcal{P}(\sigma)$ is an infinite set for each $\sigma \in \operatorname{Gal}(\mathcal{F} / \mathbb{Q})$.

\subsection{Arithmetic perturbative expansion}

We calculate the arithmetic perturbative expansion of $\tau_{p}^{S O(3)}(M)$ under the following assumption; this is a technical assumption (see Remark 2.3). 
Assumption 2.1 $M$ is a 3-manifold obtained from $S^{3}$ by 0 surgery along a knot $K$.

In this case, as mentioned before, $\tau_{p}^{S O(3)}(M) \in \mathbb{Z}_{(p)}[\zeta]$. We expand this as a polynomial in $(\zeta-1)$,

$$
\tau_{p}^{S O(3)}(M)=\sum_{\ell=0}^{p-2} \hat{\lambda}_{p, \ell}(M)(\zeta-1)^{\ell}+O\left((\zeta-1)^{p-1} ; \mathbb{Z}_{(p)}[\zeta]\right)
$$

for some $\hat{\lambda}_{p, \ell}(M)$. This expansion is not unique, but $\left(\hat{\lambda}_{p, \ell}(M)\right.$ modulo $\left.p\right)$ is uniquely determined from $\tau_{p}^{S O(3)}(M)$, so we regard $\hat{\lambda}_{p, \ell}(M)$ as in the prime field $\mathbb{F}_{p}$. Further, by (2),

$$
\hat{\lambda}_{p, \ell}(M) \underset{(p)}{\equiv} \sum_{c}\left(\frac{1+c}{1-c}\right)^{p-1} \lambda_{\ell}(M ; c) \in \mathbb{Z}_{(p)}
$$

for all but finitely many primes $p$ (to be precise, for an odd prime $p$ such that $p>\ell+1$ and $p>1+\operatorname{deg} P_{k}(t)-(2 k+1) \operatorname{deg} \Delta(t)$ for any $k \leq \ell$ and $\Delta(1)$ is not divisible by $p)$. For $\sigma \in \operatorname{Gal}(\mathcal{F} / \mathbb{Q})$, we put

$$
\tau(M ; \sigma)=\sum_{c=0, c_{1}, \ldots, c_{n}} \frac{1+\sigma(c)}{1-\sigma(c)} \cdot \frac{1-c}{1+c} \tau(M ; c) \in \mathcal{F} \llbracket q-1 \rrbracket \subset \mathbb{C} \llbracket q-1 \rrbracket,
$$

where $c_{1}, c_{1}^{-1}, \ldots, c_{n}, c_{n}^{-1}$ are the zeros of $\Delta(t)$. In particular, its $\ell$ th coefficient is given by

$$
\lambda_{\ell}(M ; \sigma)=\sum_{c=0, c_{1}, \ldots, c_{n}} \frac{1+\sigma(c)}{1-\sigma(c)} \cdot \frac{1-c}{1+c} \lambda_{\ell}(M ; c) \in \mathcal{F} \subset \mathbb{C} .
$$

Let $\mathcal{O}_{(\mathfrak{p})}$ denote the subring of $\mathcal{F}$ consisting of elements of the form $a / b$ for $a \in \mathcal{O}$ and $b \in \mathcal{O}-\mathfrak{p}$. Then, $\lambda_{\ell}(M ; \sigma) \in \mathcal{O}_{(\mathfrak{p})}$ for $\mathfrak{p}$ with $\Delta(1) \notin \mathfrak{p}$.

Proposition 2.2 Under Assumption 2.1, for all but finitely many prime ideals $\mathfrak{p} \in$ $\mathcal{P}(\sigma)$,

$$
\hat{\lambda}_{p, \ell}(M) \underset{(\mathfrak{p})}{\equiv} \lambda_{\ell}(M ; \sigma) \in \mathcal{O}_{(\mathfrak{p})}
$$

where $p$ is the prime given by $\mathfrak{p} \cap \mathbb{Z}=(p)$. Hence, the value of $\lambda_{\ell}(M ; \sigma) \in \mathcal{F}$ is uniquely determined by the series $\left(\hat{\lambda}_{p, \ell}(M)\right)_{p \text { : primes }}$ since $\mathcal{P}(\sigma)$ is an infinite set.

A more concrete assumption of $\mathfrak{p} \in \mathcal{P}(\sigma)$ for the proposition is that $\Delta(1) \notin \mathfrak{p}$, and $p>\ell+1$, and $p>1+\operatorname{deg} P_{k}(t)-(2 k+1) \operatorname{deg} \Delta(t)$ for any $k \leq \ell$. By the proposition, we can regard $\tau(M ; \sigma)$ as an arithmetic perturbative expansion of $\tau_{p}^{S O(3)}(M)$ for such a subsequence of $\mathfrak{p} \in \mathcal{P}(\sigma)$. 
Proof of Proposition 2.2 Since $\mathfrak{p} \in \mathcal{P}(\sigma)$,

$$
\left(\frac{1+c}{1-c}\right)^{p}=\operatorname{Fr}_{\mathfrak{p}}\left(\frac{1+c}{1-c}\right)=\sigma\left(\frac{1+c}{1-c}\right)=\frac{1+\sigma(c)}{1-\sigma(c)}
$$

Hence, by (6) and (7), we obtain the required formula.

Remark 2.3 Rozansky informed the author that we can show the loop expansion (1) of the colored Jones polynomial with integer coefficients for knots in integral homology 3-spheres; see Rozansky [36; 37]. If we show the above procedure using this, Assumption 2.1 can be replaced with the assumption that $H_{1}(M ; \mathbb{Z}) \cong \mathbb{Z}$.

In the following of this section, we explain how to describe the series $\left(\hat{\lambda}_{p, \ell}(M)\right)_{p \text { : primes }}$ under Assumption 2.1 and, for simplicity, the following assumption.

Assumption 2.4 The Alexander polynomial $\Delta(t)$ of $M$ does not have a multiple zero.

From (6) and the definition of $\lambda_{\ell}(M ; c)$, we have that

$$
\hat{\lambda}_{p, \ell}(M) \underset{(p)}{\equiv} \lambda_{\ell}(M ; 0)-\frac{1}{2} \sum_{i=1}^{n}\left(\frac{1+c_{i}}{1-c_{i}}\right)^{p} \operatorname{Res}_{t=c_{i}} \frac{\left(1-t^{-1}\right)^{2} P_{\ell}(t)}{\Delta(t)^{2 \ell+1}}
$$

for all but finitely many primes $p$, where we put $\Delta(t)=\prod_{i=1}^{n}\left(1-\alpha_{i}\left(t+t^{-1}-2\right)\right)$ with distinct $\alpha_{i} \in \mathbb{C}$, and $c_{i}$ is a zero of $1-\alpha_{i}\left(t+t^{-1}-2\right)$. Hence, $\hat{\lambda}_{p, \ell}(M)$ can be presented by a $\mathbb{Q}$-linear sum of 1 and

$$
\sum_{i=1}^{n}\left(\frac{1+c_{i}}{1-c_{i}}\right)^{p} \operatorname{Res}_{t=c_{i}} \frac{f_{m}\left(\alpha_{i}\right)}{t\left(1-\alpha_{i}\left(t+t^{-1}-2\right)\right)^{m}}
$$

for some polynomials $f_{m}(\alpha) \in \mathbb{Q}[\alpha]$, because, for example, putting $z=t+t^{-1}-2$,

$$
\begin{aligned}
\frac{1}{\Delta(t)} & =\sum_{i=1}^{n} \frac{\alpha_{i}^{n-1}}{\prod_{j \neq i}\left(\alpha_{i}-\alpha_{j}\right)} \frac{1}{1-\alpha_{i} z} \\
& =\frac{1}{d} \sum_{i=1}^{n} \alpha_{i}^{n-1} \prod_{j \neq i}\left(\alpha_{i}-\alpha_{j}\right) \prod_{\substack{j, j^{\prime} \neq i \\
j \neq j^{\prime}}}\left(\alpha_{j}-\alpha_{j^{\prime}}\right)^{2} \frac{1}{1-\alpha_{i} z}=\sum_{i=1}^{n} \frac{f\left(\alpha_{i}\right)}{1-\alpha_{i} z}
\end{aligned}
$$

for some polynomial $f(\alpha) \in \mathbb{Q}[\alpha]$, where $d=\prod_{i \neq j}\left(\alpha_{i}-\alpha_{j}\right)^{2} \in \mathbb{Q}$, since a symmetric polynomial in $\alpha_{j} \mathrm{~s}(j \neq i)$ can be presented by a polynomial in $\alpha_{i}$ with rational 
coefficients. Therefore, in the same way as in the proof of Proposition 1.6, $\hat{\lambda}_{p, \ell}(M)$ can be presented by a $\mathbb{Q}$-linear sum of 1 and

$$
\sum_{i=1}^{n}\left(\frac{1+c_{i}}{1-c_{i}}\right)^{p-1} g\left(\alpha_{i}\right)
$$

for some polynomial $g(\alpha) \in \mathbb{Q}[\alpha]$. Putting $\epsilon_{i}=\left(1+c_{i}\right) /\left(1-c_{i}\right)$ (and, hence, $4 \alpha_{i}+$ $\left.1=\epsilon_{i}^{2}\right), \hat{\lambda}_{p, \ell}(M)$ can be presented by a $\mathbb{Q}$-linear sum of 1 and $\sum_{i=1}^{n} \epsilon_{i}^{p+k}$ for $k=-1,1,3, \ldots$ Further, we note that $\epsilon_{1}^{2}, \ldots, \epsilon_{n}^{2}$ equals the zeros of $F(x)$, where we set $\nabla(z) \in \mathbb{Q}[z]$ by $\Delta(t)=\nabla(z)=\prod_{i}\left(1-\alpha_{i} z\right)$ putting $z=t+t^{-1}-2$, and set $F(x) \in \mathbb{Q}[x]$ by $F(x)=z^{n} \nabla(1 / z)=\prod_{i}\left(z-\alpha_{i}\right)$ putting $x=4 z+1$. Since $F(x)$ is a polynomial of degree $n, \sum_{i=1}^{n} \epsilon_{i}^{p+k}$ for each odd $k$ can be presented by a $\mathbb{Q}$-linear sum of $\sum_{i=1}^{n} \epsilon_{i}^{p+b}$ for $b=1,3, \ldots, 2 n-1$. Therefore, $\hat{\lambda}_{p, \ell}(M)$ can be presented by a $\mathbb{Q}$-linear sum of 1 and $\sum_{i=1}^{n} \epsilon_{i}^{p+b}$ for $b=1,3, \ldots, 2 n-1$.

Let us regard the series $\left(\hat{\lambda}_{p, \ell}(M)\right)_{p \text { : primes }}$ as in

$$
\mathbb{F}=\prod_{\text {primes } p} \mathbb{F}_{p} / \bigoplus_{\text {primes } p} \mathbb{F}_{p}
$$

that is, we consider the series $\left(\hat{\lambda}_{p, \ell}(M)\right)_{p \text { : primes }} \in \prod_{\text {primes }} \mathbb{F}_{p}$ modulo the equivalence that $\left(a_{p}\right) \sim\left(a_{p}^{\prime}\right)$ when $a_{p}=a_{p}^{\prime}$ for all but finitely many primes $p$. Note that $\mathbb{F}$ forms a $\mathbb{Q}$-algebra. In $\mathbb{F}$, the series $\left(\hat{\lambda}_{p, \ell}(M)\right)_{p \text { :primes }}$ can be presented by a linear sum of 1 and $\left(\sum_{i} \epsilon_{i}^{p+b}\right)_{p \text { : primes }}$ for $b=1,3, \ldots, 2 n-1$.

Further, by the Tamagawa map given in Section 2.2, such a linear sum in $\mathbb{F}$ is identified with an element in $\mathbb{C} \otimes \mathbb{Q} \mathbb{C}$, and, in particular, the Tamagawa map takes $\left(\hat{\lambda}_{p, \ell}(M)\right)_{p \text { : primes }}$ to

$-\frac{1}{2} \sum_{c=0, c_{1}, \ldots, c_{n}} \frac{1+c}{1-c} \otimes \operatorname{Res}_{t=c} \frac{\left(1-t^{-1}\right)^{2} P_{\ell}(t)}{\Delta(t)^{2 \ell+1}}=\sum_{c=0, c_{1}, \ldots, c_{n}} \frac{1+c}{1-c} \otimes \frac{1-c}{1+c} \lambda_{\ell}(M ; c) \in \mathbb{C} \otimes_{\mathbb{Q}} \mathbb{C}$.

So, we put

$$
\tau(M)=\sum_{c=0, c_{1}, \ldots, c_{n}} \frac{1+c}{1-c} \otimes \frac{1-c}{1+c} \tau(M ; c) \in\left(\mathbb{C} \otimes_{\mathbb{Q}} \mathbb{C}\right) \llbracket q-1 \rrbracket .
$$

It can be shown from the topological invariance of $\tau_{p}^{S O(3)}(M)$ (without using Theorem 1.1) that this is a topological invariant of $M$, when $M$ satisfies Assumptions 2.1 and 2.4. 
As mentioned before, $\tau(M ; \sigma)$ can be regarded as an arithmetic perturbative expansion of $\tau_{p}^{S O(3)}(M)$ for a subsequence $\mathcal{P}(\sigma)$. The vector $(\tau(M ; \sigma))_{\sigma}$ is taken to $\tau(M)$, by the inverse matrix of the matrix $\left(\sigma\left(f_{i}\right)\right)_{\sigma, i}$ given in the proof of Lemma 2.6. Hence, $\tau(M)$ is an invariant obtained from the vector $(\tau(M ; \sigma))_{\sigma}$ by such a coordinate change that the resulting $\tau(M)$ does not depend on the Galois group.

\subsection{The Tamagawa map}

The arguments of this subsection are due to Akio Tamagawa. For the preliminary of number theory used in this subsection, see, for example, Neukirch [24] and Ribenboim [35].

Let $F(x)$ and $\epsilon_{1}, \ldots, \epsilon_{n}$ be as above. We use the notation given at the beginning of this section.

Proposition 2.5 (A Tamagawa) The following map

$$
\left(\sum_{i=1}^{n} \epsilon_{i}^{a p+b}\right)_{p: \text { primes }} \longmapsto \sum_{i=1}^{n} \epsilon_{i}^{a} \otimes \epsilon_{i}^{b} \in \mathcal{F} \otimes_{\mathbb{Q}} \mathcal{F} \subset \mathbb{C} \otimes_{\mathbb{Q}} \mathbb{C}
$$

induces an isomorphism between algebras,

$$
\begin{aligned}
& \operatorname{span}_{\mathbb{Q}}\left\{\left(\sum_{i=1}^{n} \epsilon_{i}^{a p+b}\right)_{p} \mid a+b \text { is even }\right\} \cong \operatorname{span}_{\mathbb{Q}}\left\{\sum_{i=1}^{n} \epsilon_{i}^{a} \otimes \epsilon_{i}^{b} \mid a+b \text { is even }\right\} . \\
& \text { F } \\
& \mathcal{F} \otimes_{\mathbb{Q}} \mathcal{F}
\end{aligned}
$$

Recall that $k_{\mathfrak{p}}$ is the residue field $\mathcal{O} / \mathfrak{p}$ for a prime ideal $\mathfrak{p} \subset \mathcal{O}$. Similarly as $\mathbb{F}$ is a $\mathbb{Q}$-algebra, $\prod_{\mathfrak{p}} k_{\mathfrak{p}} / \bigoplus_{\mathfrak{p}} k_{\mathfrak{p}}$ forms an $\mathcal{F}$-algebra, where $\mathfrak{p}$ runs over all prime ideals of $\mathcal{O}$ in the direct product and in the direct sum; we can further assume that $\mathfrak{p} \in \mathcal{P}$, since we can ignore finitely many $\mathfrak{p}$ in $\prod_{\mathfrak{p}} k_{\mathfrak{p}} / \bigoplus_{\mathfrak{p}} k_{\mathfrak{p}}$.

Proof of Proposition 2.5 We consider the following homomorphisms,

$$
\mathbb{F} \longrightarrow \prod_{\mathfrak{p}} k_{\mathfrak{p}} / \bigoplus_{\mathfrak{p}} k_{\mathfrak{p}} \stackrel{\iota}{\longleftarrow} \mathcal{F} \otimes_{\mathbb{Q}} \mathcal{F}
$$

where the left homomorphism is the natural homomorphism induced by the inclusion $\mathbb{F}_{p} \rightarrow k_{\mathfrak{p}}$, and the right homomorphism $\iota$ is defined by $\iota(\epsilon \otimes \delta)=\left(\epsilon^{p} \delta\right)_{\mathfrak{p} \in \mathcal{P}}$. By definition, the images of

$$
\left(\sum_{i=1}^{n} \epsilon_{i}^{a p+b}\right)_{p: \text { primes }} \text { and } \sum_{i=1}^{n} \epsilon_{i}^{a} \otimes \epsilon_{i}^{b}
$$


in $\prod_{\mathfrak{p}} k_{\mathfrak{p}} / \bigoplus_{\mathfrak{p}} k_{\mathfrak{p}}$ are equal. Further, since the homomorphism $\iota$ is injective by Lemma 2.6 below, the homomorphism (10) is well defined.

Moreover, since the inverse homomorphism of (11) is well defined, (11) is an isomorphism.

Lemma 2.6 (A Tamagawa) The homomorphism

$$
\iota: \mathcal{F} \otimes_{\mathbb{Q}} \mathcal{F} \longrightarrow \prod_{\mathfrak{p}} k_{\mathfrak{p}} / \bigoplus_{\mathfrak{p}} k_{\mathfrak{p}}
$$

given by $\iota(\epsilon \otimes \delta)=\left(\epsilon^{p} \delta\right)_{\mathfrak{p} \in \mathcal{P}}$ is an injective $\mathcal{F}$-homomorphism, where we regard $\mathcal{F} \otimes_{\mathbb{Q}} \mathcal{F}$ as an $\mathcal{F}$-algebra by the multiplication of a scalar $\in \mathcal{F}$ to the second factor of $\mathcal{F} \otimes_{\mathbb{Q}} \mathcal{F}$.

Proof Since $\mathcal{P}$ splits into the disjoint union of $\mathcal{P}(\sigma) \mathrm{s}$, we have that

$$
\prod_{\mathfrak{p}} k_{\mathfrak{p}} / \bigoplus_{\mathfrak{p}} k_{\mathfrak{p}}=\bigoplus_{\sigma}\left(\prod_{\mathfrak{p} \in \mathcal{P}(\sigma)} k_{\mathfrak{p}} / \bigoplus_{\mathfrak{p} \in \mathcal{P}(\sigma)} k_{\mathfrak{p}}\right) .
$$

We consider the map

$$
\mathcal{F} \longrightarrow \prod_{\mathfrak{p} \in \mathcal{P}(\sigma)} k_{\mathfrak{p}} / \bigoplus_{\mathfrak{p} \in \mathcal{P}(\sigma)} k_{\mathfrak{p}}
$$

induced by the natural map $\mathcal{O}_{(\mathfrak{p})} \rightarrow k_{\mathfrak{p}}$. Since $\mathcal{P}(\sigma)$ is an infinite set, this map is injective. By making the direct sum of such maps, we have an injective linear map.

$$
\mathcal{F}^{\oplus m} \longrightarrow \prod_{\mathfrak{p}} k_{\mathfrak{p}} / \bigoplus_{\mathfrak{p}} k_{\mathfrak{p}},
$$

where $m$ is the degree of the extension $\mathcal{F} / \mathbb{Q}$, which is equal to the cardinality of $\operatorname{Gal}(\mathcal{F} / \mathbb{Q})$. This linear map takes the natural basis of $\mathcal{F}^{\oplus m}$ to

$$
e_{\sigma}=\left(e_{\sigma, \mathfrak{p}}\right)_{\mathfrak{p} \in \mathcal{P}}, \quad e_{\sigma, \mathfrak{p}}= \begin{cases}1 & \text { if } \mathfrak{p} \in \mathcal{P}(\sigma), \\ 0 & \text { otherwise }\end{cases}
$$

In terms of $e_{\sigma}$, the linear map

$$
\iota: \mathcal{F} \otimes_{\mathbb{Q}} \mathcal{F} \longrightarrow \mathcal{F}^{\oplus m} \subset \prod_{\mathfrak{p}} k_{\mathfrak{p}} / \bigoplus_{\mathfrak{p}} k_{\mathfrak{p}}
$$

is presented by

$$
\iota(\epsilon \otimes \delta)=\left(\epsilon^{p} \delta\right)_{\mathfrak{p} \in \mathcal{P}}=\sum_{\sigma} \operatorname{Fr}_{\mathfrak{p}}(\epsilon) \delta e_{\sigma}=\sum_{\sigma} \sigma(\epsilon) \delta e_{\sigma}
$$


For an integral basis $\left\{f_{i}\right\}$ of $\mathcal{O}$, this linear map is presented by the matrix $\left(\sigma\left(f_{i}\right)\right)_{\sigma, i}$. Its determinant is the discriminant of the extension $\mathcal{F} / \mathbb{Q}$, and is non-zero. Hence, $\iota$ is an injective $\mathcal{F}$-linear map. Further, since $\iota$ is a homomorphism by definition, $\iota$ is an injective $\mathcal{F}$-homomorphism.

Remark 2.7 This remark is on a personal history of the research of this paper. In an early stage of the research, the author observed that the arithmetic perturbative expansion of $\tau_{p}^{S O(3)}(M)$ can be presented by a linear sum of $1, \gamma_{0}, \ldots, \gamma_{n-1}$ where

$$
\gamma_{j}=\sum_{i=1}^{n} \epsilon_{i}^{p+2 j-1} \quad \text { and } \quad \epsilon_{i}=\left(1+c_{i}\right) /\left(1-c_{i}\right)
$$

for the zeros $c_{1}^{ \pm 1}, \ldots, c_{n}^{ \pm 1}$ of $\Delta(t)$, and obtained results such as those in Sections 2.3 and 2.4, when the irreducible factors of the Alexander polynomial $\Delta(t)$ is of degree $\leq 2$. In order to obtain results when irreducible factors of $\Delta(t)$ is of general degree, it was a problem to describe the space $W=\operatorname{span}_{\mathbb{Q}}\left\{1, \gamma_{0}, \ldots, \gamma_{n-1}\right\}$. The author asked Akio Tamagawa how to describe $W$, and Tamagawa soon constructed the map (11) for $a=1$. Motivated by this map, the author presented the coefficients of the linear sum in terms of integral, showing Proposition 6.1, and observed that the image of the linear sum by the Tamagawa map (11) is presented by the tensor product (8) for the $\ell$ th coefficient of the arithmetic perturbative expansion of $\tau_{p}^{S O(3)}(M)$. After that, the author directly proved Theorem 1.1, which implies that the second factor of this tensor product is also a topological invariant, by introducing the $t$-through relation.

\subsection{The case where $\Delta(t)$ is of degree 0}

In this case, the arithmetic perturbative expansion of $\tau_{p}^{S O(3)}(M)$ is described (without using the Tamagawa map), as follows.

As a particular case of (6), we have

Proposition 2.8 Under Assumption 2.1, for all but finitely many primes $p$,

$$
\hat{\lambda}_{p, \ell}(M) \underset{(p)}{\equiv} \lambda_{\ell}(M ; 0) .
$$

That is, for such $p$,

$$
\tau_{p}^{S O(3)}(M)=\tau(M ; 0)^{(\leq \ell)}+O\left((\zeta-1)^{\ell+1} ; \mathbb{Z}_{(p)}[\zeta]\right),
$$

where we put $\tau(M ; \cdot)^{(\leq \ell)}=\sum_{k=0}^{\ell} \lambda_{k}(M ; \cdot)(q-1)^{k}$. 
A more concrete assumption of $p$ for the proposition is that $b_{0}$ is not divisible by $p$ and $p>\ell+1$ and $p>1+\operatorname{deg} P_{k}(t)$ for any $k \leq \ell$.

Therefore, as an alternative form of $(9), \tau(M)=1 \otimes \tau(M ; 0)$ is regarded as the arithmetic perturbative expansion of $\tau_{p}^{S O(3)}(M)$.

\subsection{The case where the irreducible factors of $\Delta(t)$ are of degree 1}

In this case, the arithmetic perturbative expansion of $\tau_{p}^{S O(3)}(M)$ is described, as follows.

Proposition 2.9 Let $M$ be a 3-manifold whose Alexander polynomial is presented by $\Delta(t)=\prod_{i=n}^{n}\left(1-a_{i}\left(t+t^{-1}-2\right)\right)$ with $a_{i} \in \mathbb{Q}$, satisfying Assumptions 2.1 and 2.4. Then, for all but finitely many primes $p$,

$$
\hat{\lambda}_{p, \ell}(M) \underset{(p)}{\equiv} \lambda_{\ell}(M ; 0)+\sum_{i=1}^{n}\left(\frac{4 a_{i}+1}{p}\right) \lambda_{\ell}\left(M ; c_{i}\right),
$$

where $c_{i}$ is a zero of $1-a_{i}\left(t+t^{-1}-2\right)$, and $(\dot{\dot{p}})$ denotes the Legendre symbol. That is, for such $p$,

$$
\tau_{p}^{S O(3)}(M)=\tau(M ; 0)^{(\leq \ell)}+\sum_{i=1}^{n}\left(\frac{4 a_{i}+1}{p}\right) \tau\left(M ; c_{i}\right)^{(\leq \ell)}+O\left((\zeta-1)^{\ell+1} ; \mathbb{Z}_{(p)}[\zeta]\right) .
$$

A more concrete assumption of $p$ for the proposition is that $\Delta( \pm 1)$ are not divisible by $p$ and $p>\ell+1$ and $p>\operatorname{deg} P_{k}(t)-2 \ell$ for any $k \leq \ell$.

Proof of Proposition 2.9 By (6),

$$
\hat{\lambda}_{p, \ell}(M) \underset{(p)}{\equiv} \lambda_{\ell}(M ; 0)+\sum_{i=1}^{n}\left(\frac{1+c_{i}}{1-c_{i}}\right)^{p-1} \lambda_{\ell}\left(M ; c_{i}\right)
$$

Since $1-a_{i}\left(c_{i}+c_{i}^{-1}-2\right)=0$, we have that $c_{i}+c_{i}^{-1}-2=a_{i}^{-1}$. Hence,

$$
\begin{aligned}
\left(\frac{1+c_{i}}{1-c_{i}}\right)^{2} & =\frac{a_{i}^{-1}+4}{a_{i}^{-1}}=4 a_{i}+1 \\
\text { and } \quad\left(\frac{1+c_{i}}{1-c_{i}}\right)^{p-1} & =\left(4 a_{i}+1\right)^{(p-1) / 2} \underset{(p)}{\equiv}\left(\frac{4 a_{i}+1}{p}\right) .
\end{aligned}
$$

By substituting this formula to the above formula of $\hat{\lambda}_{p, \ell}(M)$, we obtain the required formula. 
We put

$$
\varepsilon_{k}=\left(\left(\frac{k}{2}\right),\left(\frac{k}{3}\right),\left(\frac{k}{5}\right), \ldots\right) \in \mathbb{F},
$$

and denote by $\mathcal{R}_{1}$ the subring of $\mathbb{F}$ generated by $\varepsilon_{k}$ for non-zero integers $k$, ignoring primes $p$ for which the Legendre symbol $\left(\frac{k}{p}\right)$ is not defined. Then,

$$
\mathcal{R}_{1}=\mathbb{Q}\left[\varepsilon_{-1}, \varepsilon_{2}, \varepsilon_{3}, \varepsilon_{5}, \ldots\right] /\left(\varepsilon_{-1}^{2}=\varepsilon_{2}^{2}=\varepsilon_{3}^{2}=\varepsilon_{5}^{2}=\cdots=1\right)
$$

by the Dirichlet prime number theorem which implies that there are infinitely many primes with same values for Legendre symbols. We can verify that the relations among $\varepsilon_{k}$ in (12) are sufficient, since $\mathcal{R}_{1}$ is embedded into $\mathbb{C} \otimes \mathbb{Q} \mathbb{C}$ by the Tamagawa map (11),

$$
\mathcal{R}_{1} \longrightarrow \mathbb{C} \otimes \mathbb{Q} \mathbb{C}, \quad \varepsilon_{k} \longmapsto \sqrt{k} \otimes \frac{1}{\sqrt{k}},
$$

which is an algebra homomorphism in this domain. Therefore, as an alternative form of (9),

$$
\tau(M ; 0)+\sum_{i=1}^{n} \varepsilon_{4 a_{i}+1} \tau\left(M ; c_{i}\right) \in \mathcal{R}_{1} \llbracket q-1 \rrbracket
$$

is regarded as the arithmetic perturbative expansion of $\tau_{p}^{S O(3)}(M)$.

\section{The loop expansion}

In this section, we review the loop expansions of the Kontsevich invariant and the colored Jones polynomials in Section 3.1 and 3.2 respectively. The latter can be obtained from the former by $\mathfrak{s l}_{2}$ reduction; we explain its concrete procedure in Section 3.2.

\subsection{The loop expansion of the Kontsevich invariant}

In this subsection, we review the loop expansion of the Kontsevich invariant and how we obtain it by the rational version of the Aarhus integral.

The Kontsevich invariant is defined in the space of Jacobi diagrams on $S^{1}$, which we define as follows. For a 1 -manifold $X$, a Jacobi diagram on $X$ is the manifold $X$ together with a uni-trivalent graph such that univalent vertices of the graph are distinct points on $X$ and each trivalent vertex is vertex-oriented, where a vertex-oriented trivalent vertex is a trivalent vertex such that a cyclic order of the three edges around the trivalent vertex is fixed. In figures we draw $X$ by thick lines and the uni-trivalent graphs by thin lines, in such a way that each trivalent vertex is vertex-oriented in the counterclockwise order. We define the degree of a Jacobi diagram to be half the number 
of univalent and trivalent vertices of the uni-trivalent graph of the Jacobi diagram. We denote by $\mathcal{A}(X)$ the quotient vector space spanned by Jacobi diagrams on $X$ subject to the following relations, called the $A S, I H X$, and STU relations respectively,
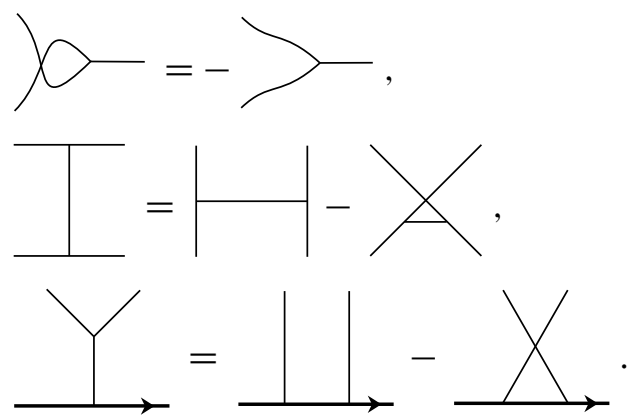

The Kontsevich invariant $Z(K)$ [16] of a knot $K$ is defined to be in $\mathcal{A}\left(S^{1}\right)$; for details of its constructions see, for example, the author's book [28].

The loop expansion of the Kontsevich invariant is defined in the space of open Jacobi diagrams. An open Jacobi diagram is a vertex-oriented uni-trivalent graph. We denote by $\mathcal{A}(*)$ the quotient vector space spanned by open Jacobi diagrams subject to the AS and IHX relations. The Poincare-Birkhoff-Witt isomorphism $\chi: \mathcal{A}(*) \rightarrow \mathcal{A}(\downarrow)$ is defined by

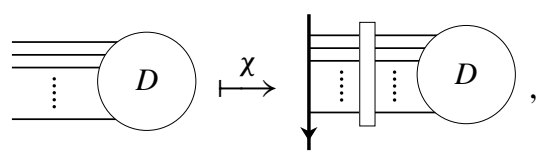

for any diagram $D$, where the box denotes the symmetrizer

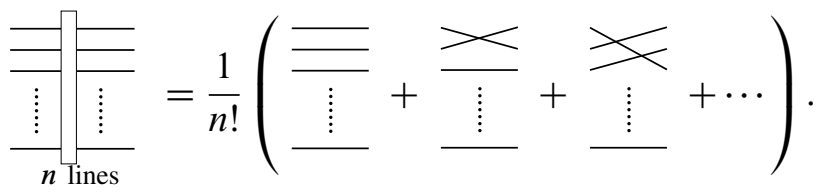

A label of a power series $f(\hbar)=c_{0}+c_{1} \hbar+c_{2} \hbar^{2}+c_{3} \hbar^{3}+\cdots$ implies that

$$
\left.\stackrel{f(\hbar)}{=} c_{0}\right)+c_{1} \sum^{\hbar}+c_{2} \sum^{\hbar}+c_{3} \sum_{\hbar}^{\hbar}+\cdots .
$$

Note that

$$
f(\hbar)|=| f(-\hbar)
$$


by the AS relation, in the notation of this paper. Any open Jacobi diagram can be presented by a trivalent graph with labels on its edges. It is known (see Rozansky [42], Kricker [17] and Garoufalidis-Kricker [9]) that the Kontsevich invariant of a knot $K$ has a presentation, called the loop expansion,

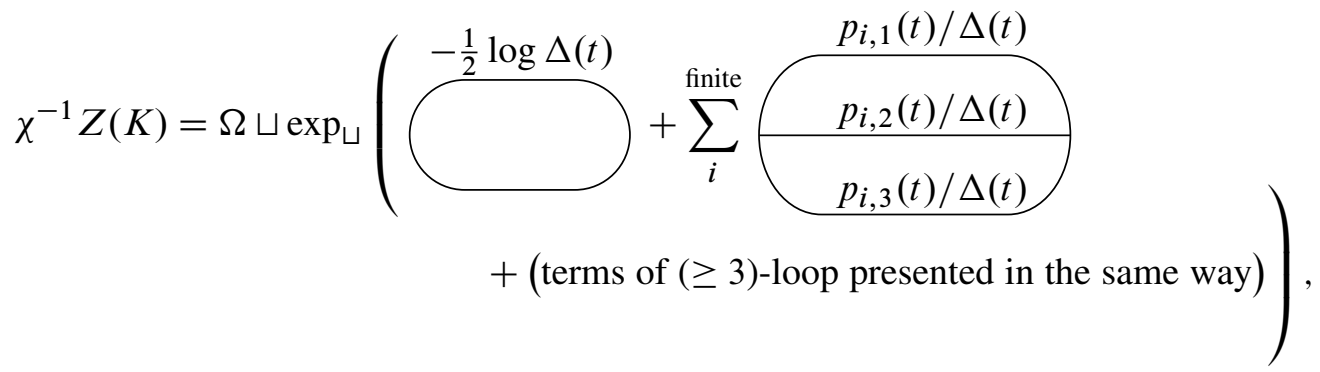

where we put $t=e^{\hbar}$, and $\exp _{\sqcup}$ denotes the exponential with respect to the disjointunion product of open Jacobi diagrams, $\Delta(t)$ denotes the Alexander polynomial of $K$, and $p_{i, j}(t)$ is a polynomial in $t^{ \pm 1}$, and we put

$$
\Omega=\chi^{-1} Z(\text { the trivial knot })=\exp _{\sqcup}(\underbrace{\frac{1}{2} \log \frac{\sinh (\hbar / 2)}{\hbar / 2}}) \text {. }
$$

Similarly, it can be shown that the LMO invariant of a pair of a rational homology 3-sphere $N$ and a null-homologous knot $K$ in $N$ is also presented by the loop expansion,

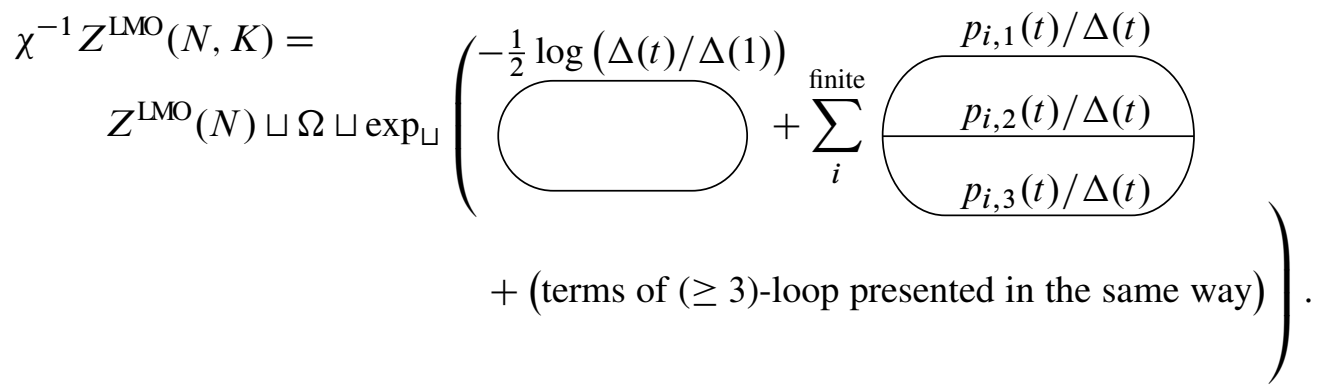

We briefly review how we obtain the loop expansion; for detailed and precise arguments, see Kricker [17] and Garoufalidis-Kricker [9]. The Kontsevich invariant of the long Hopf link is presented by

$$
\chi^{-1} Z\left(\frac{\downarrow-|\ldots|-\uparrow \ldots}{\downarrow \downarrow}\right)=\downarrow t \mid t \ldots \uparrow t^{-1} \uparrow t^{-1} \sqcup \Omega,
$$


where we put $t=e^{\hbar}$. A pair of a rational homology 3-sphere $N$ and a null-homologous knot $K$ in $N$ can be obtained from $\left(S^{3}, K_{0}\right)$ by surgery along $K$ for some framed link $K_{0} \cup L$ in $S^{3}$ such that $K_{0}$ is the trivial knot and each component of $L$ is null-homologous in the complement of $K_{0}$. Let $A$ be the equivariant linking matrix (see Kojima-Yamasaki [15] and Garoufalidis-Kricker [7]) of a lift of $L$ in the infinite cyclic cover of $S^{3}-K_{0}$; its entries are in $\mathbb{Z}\left[t^{ \pm 1}\right]$. Then, the Kontsevich invariant of $K_{0} \cup L$ can be presented by the form

$$
\chi^{-1} Z\left(K_{0} \cup L\right)=\Omega \sqcup \exp \left(\frac{1}{2} \sum_{i, j} \bigcap_{y_{i}}^{A_{i j}}\right) \sqcup R,
$$

where $R$ is a linear sum of open Jacobi diagrams with labels of polynomials in $t^{ \pm 1}$ and with at least 3 univalent vertices; this presentation follows from (16). The rational version of the Aarhus integral takes it to

$$
\begin{aligned}
& \chi^{-1} Z^{\mathrm{LMO}}(N, K)=c_{+}^{-\sigma_{+}} c_{-}^{-\sigma_{-}} \int^{\mathrm{rat}} \chi^{-1} Z\left(K_{0} \cup L\right) d Y= \\
& c_{+}^{-\sigma_{+}} c_{-}^{-\sigma_{-}} \sqcup \Omega \sqcup \exp _{\sqcup}(\overbrace{}^{-\frac{1}{2} \log (\Delta(t) / \Delta(1))}) \sqcup\left\langle\exp (-\frac{1}{2} \sum_{i, j} \underbrace{\partial y_{j}}_{\left(A^{-1}\right)_{i j}} \int^{\partial y_{i}}), R\right\rangle,
\end{aligned}
$$

where $c_{ \pm} \in \mathcal{A}(\varnothing)$ are some normalization constants, $\sigma_{ \pm}$are the number of positive and negative eigenvalues of the linking matrix of $L$, and the bracket is defined by

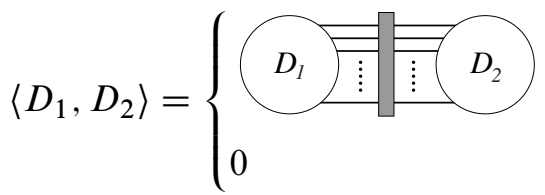

$$
\begin{aligned}
& \text { if the number of } \partial y_{i}-\text { legs of } D_{1} \text { equals } \\
& \text { the number of } y_{i} \text {-legs of } D_{2} \text { for each } i \text {, } \\
& \text { otherwise, }
\end{aligned}
$$

for open Jacobi diagrams $D_{1}$ with $\partial y_{i}$-legs and $D_{2}$ with $y_{i}$-legs. Here, a shaded box means the sum of all ways of connecting the left lines to the right lines, which is equal to $n$ ! times (14).

\subsection{The loop expansion of the colored Jones polynomial}

In this subsection, we explain the loop expansion of the colored Jones polynomial (Proposition 3.1 and Remark 3.2) is obtained from the loop expansion of the Kontsevich invariant by $\mathfrak{s l}_{2}$ reduction. 
The $\mathfrak{s l}_{2}$ reduction is a procedure reducing an open Jacobi diagram with labels of rational functions to a rational function, in the following way. We resolve trivalent vertices by

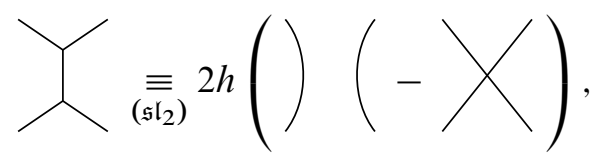

where $h$ is a variable satisfying that $q=e^{h}$, noting that the $\mathfrak{s l}_{2}$ weight system of both sides are equal; see Chmutov-Varchenko [4] and the author's book [28]. By using this formula recursively, we have that

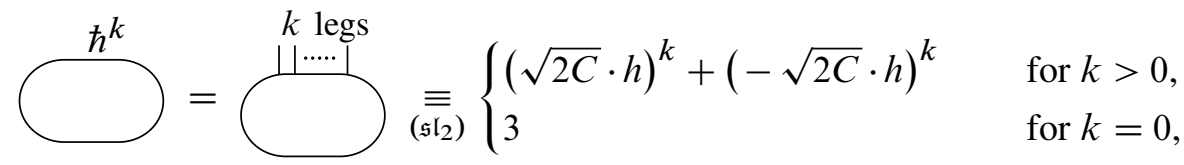

where $C$ denotes the diagram consisting of a single arc. Hence, for a function $\varphi(t)$ which has an expansion $\varphi\left(e^{\hbar}\right) \in \mathbb{Q} \llbracket \hbar \rrbracket$,

$$
\Upsilon^{\varphi(t)} \underset{\left(\mathfrak{s l}_{2}\right)}{\equiv} \varphi(\hat{t})+\varphi\left(\hat{t}^{-1}\right)+\varphi(1),
$$

where we put $t=e^{\hbar}$ and $\hat{t}=e^{\sqrt{2 C} \cdot h}$. In a similar way, we can also show (see the author's paper [30]) that

$$
\varphi_{\varphi_{2}(t)}^{\varphi_{1}(t)} \underset{\varphi_{3}(t)}{\equiv} 2 h \sum_{\left(\mathfrak{s l}_{2}\right)} \varphi_{i}(\hat{t}) \varphi_{j}\left(\hat{t}^{-1}\right) \varphi_{k}(1),
$$

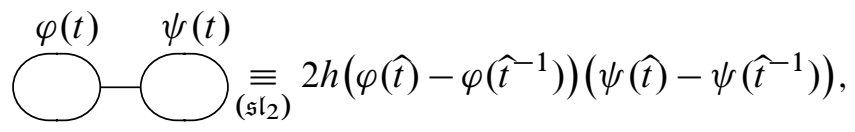

for rational functions $\varphi_{i}(t), \varphi(t)$ and $\psi(t)$. In general, we can decrease the number of trivalent vertices, as follows. Consider a trivalent vertex,

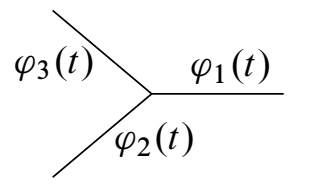

By putting $\hat{\varphi}_{i}(t)=\varphi_{i}(t)-\varphi_{i}(1)$, the above diagram is equal to the sum of diagrams obtained from it by replacing each $\varphi_{i}(t)$ with $\hat{\varphi}_{i}(t)$ or $\varphi_{i}(1)$. If we choose $\hat{\varphi}_{i}(t)$ for 
all $i$, the diagram vanishes, since

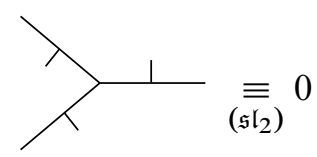

by (17). Hence, at least one of the $\varphi_{i}(t) \mathrm{s}$ is replaced with $\varphi_{i}(1)$. By applying (17) to the edge corresponding to $\varphi_{i}(1)$, we can decrease the number of trivalent vertices. In this way, we can reduce a Jacobi diagram with labels of rational functions of $t$ to a rational function of $\hat{t}$ by $\mathfrak{s l}_{2}$ reduction. Further,

$$
\exp _{\sqcup}(\overbrace{}^{-\frac{1}{2} \log (\Delta(t) / \Delta(1))}) \underset{\left(\mathfrak{s l}_{2}\right)}{\equiv} \exp (-\log (\Delta(\hat{t}) / \Delta(1)))=\frac{\Delta(1)}{\Delta(\hat{t})} .
$$

Therefore, since the $\mathfrak{s l}_{2}$ reduction of $Z^{\mathrm{LMO}}(N) \in \mathcal{A}(\varnothing)$ is given by a power series in $\mathbb{Q} \llbracket h \rrbracket \cong \mathbb{Q} \llbracket q-1 \rrbracket$, the $\mathfrak{s l}_{2}$ reduction of the loop expansion of $\chi^{-1} Z^{\mathrm{LMO}}(N, K)$ is presented by

$$
\chi^{-1} Z^{\mathrm{LMO}}(N, K) \sqcup \Omega^{-1} \underset{\left(\mathfrak{\mathfrak { s l } _ { 2 }}\right)}{\equiv} \sum_{\ell=0}^{\infty} \frac{\widehat{P}_{\ell}(\hat{t})}{\Delta\left(\hat{t}^{2 \ell+1}\right.}(q-1)^{\ell}
$$

for some symmetric polynomials $\widehat{P}_{\ell}(\hat{t}) \in \mathbb{Q}\left[\hat{t}^{ \pm 1}\right]$.

Proposition 3.1 Let $K$ be a null-homologous knot in a rational homology 3-sphere $N$. Then, $W_{\mathfrak{s l}_{2}, V_{n}}\left(Z^{\mathrm{LMO}}(N, K)\right)$ is presented by the form,

$$
W_{\mathfrak{s l}_{2}, V_{n}}\left(Z^{\mathrm{LMO}}(N, K)\right)=[n] \sum_{\ell=0}^{\infty} \frac{P_{\ell}\left(q^{n}\right)}{\Delta\left(q^{n}\right)^{2 \ell+1}}(q-1)^{\ell},
$$

for some polynomials $P_{\ell}(t) \in \mathbb{Q}\left[t^{ \pm 1}\right]$, where $\Delta(t)$ is the Alexander polynomial of $K$; in particular, $P_{0}(t)(=\Delta(1))$ is equal to the order of $H_{1}(N ; \mathbb{Z})$.

Proof Since $\chi(\Omega)$ is the Kontsevich invariant of the trivial knot, $W_{\mathfrak{s l}_{2}, V_{n}}(\chi(\Omega))=[n]$. Hence,

$$
\Omega \underset{\left(\mathfrak{s l}_{2}\right)}{\equiv} \frac{e^{\sqrt{2 C} \cdot h / 2}-e^{-\sqrt{2 C} \cdot h / 2}}{\sqrt{2 C} \cdot h} \stackrel{W_{\mathfrak{s l}_{2}, V_{n}} \circ \chi}{\longmapsto}[n]=\frac{q^{n / 2}-q^{-n / 2}}{q^{1 / 2}-q^{-1 / 2}} .
$$

By replacing $h$ with $k h$,

$$
\frac{1}{k} \cdot \frac{\hat{t}^{k / 2}-\hat{t}^{-k / 2}}{\hat{t}^{1 / 2}-\hat{t}^{-1 / 2}} \cdot \Omega \longmapsto[n] \cdot \frac{t^{k / 2}-t^{-k / 2}}{t^{1 / 2}-t^{-1 / 2}} \cdot \frac{1}{[k]},
$$


where we put $\hat{t}=e^{\sqrt{2 C} \cdot h}$ and $t=q^{n}$. Therefore,

$$
\begin{aligned}
\varphi(\hat{t}) \Omega \longmapsto[n] \cdot \frac{1}{t^{1 / 2}-t^{-1 / 2}} \cdot \frac{D_{\alpha}}{\left[D_{\alpha}\right]}\left(\left.\left(t^{1 / 2}-t^{-1 / 2}\right) \varphi(t)\right|_{t^{1 / 2} \rightarrow e^{\alpha}}\right) \\
=[n] \cdot \frac{1}{t^{1 / 2}-t^{-1 / 2}} \cdot \frac{D}{[D]}\left(\left(t^{1 / 2}-t^{-1 / 2}\right) \varphi(t)\right),
\end{aligned}
$$

where we put $D_{\alpha}=\frac{d}{d \alpha}$ and $D=2 t \frac{d}{d t}$, regarding $\frac{D}{[D]}=\frac{e^{h / 2}-e^{-h / 2}}{e^{h D / 2}-e^{-h D / 2}} D$ as a power series in $(h D)^{2}$ and $h$. Hence, we can determine $P_{\ell}(t)$ by

$$
\sum_{\ell} \frac{P_{\ell}(t)}{\Delta(t)^{2 \ell+1}}(q-1)^{\ell}=\frac{1}{t^{1 / 2}-t^{-1 / 2}} \cdot \frac{D}{[D]}\left(\left(t^{1 / 2}-t^{-1 / 2}\right) \sum_{\ell} \frac{\hat{P}_{\ell}(t)}{\Delta(t)^{2 \ell+1}}(q-1)^{\ell}\right)
$$

where we can show that $P_{\ell}(t) \in \mathbb{Q}\left[t^{ \pm 1}\right]$, since

$$
\frac{1}{t^{1 / 2}-t^{-1 / 2}} \cdot(h D)^{2 m}\left(\left(t^{1 / 2}-t^{-1 / 2}\right) \frac{\widehat{P}_{\ell}(t)}{\Delta(t)^{2 \ell+1}}(q-1)^{\ell}\right)
$$

is presented by a linear sum of the form $\frac{f_{k}(t)}{\Delta(t)^{2 k+1}}(q-1)^{k}$ for some polynomials $f_{k}(t) \in \mathbb{Q}\left[t^{ \pm 1}\right]$.

Remark 3.2 In particular, when $N=S^{3}$,

$$
W_{\mathfrak{s l}_{2}, V_{n}}\left(Z^{\mathrm{LMO}}\left(S^{3}, K\right)\right)=W_{\mathfrak{s l}_{2}, V_{n}}(Z(K))=[n] J_{n}(K ; q) .
$$

Hence, in this case, Proposition 3.1 implies the loop expansion of the colored Jones polynomial,

$$
J_{n}(K ; q)=\sum_{\ell=0}^{\infty} \frac{P_{\ell}\left(q^{n}\right)}{\Delta\left(q^{n}\right)^{2 \ell+1}}(q-1)^{\ell} .
$$

This formula was shown by Rozansky; he further shows that $P_{\ell}(t) \in \mathbb{Z}\left[t^{ \pm 1}\right]$ in [41].

Remark 3.3 In the defining relation (2) of the perturbative invariant, we can replace $P_{\ell}(t)$ with $\widehat{P}_{\ell}(t)$, since

$$
\operatorname{Res}_{t=c} \frac{\left(1-t^{-1}\right)^{2} P_{\ell}(t)}{\Delta(t)^{2 \ell+1}}=\operatorname{Res}_{t=c} \frac{\left(1-t^{-1}\right)^{2} \widehat{P}_{\ell}(t)}{\Delta(t)^{2 \ell+1}},
$$

where this equality is obtained as follows. By (4),

$$
\operatorname{Res}_{t=c} \frac{t^{1 / 2} \mp t^{-1 / 2}}{t} D \varphi(t)=-\operatorname{Res}_{t=c} \frac{t^{1 / 2} \pm t^{-1 / 2}}{t} \varphi(t)
$$


for $D=2 t \frac{d}{d t}$. Hence,

$$
\operatorname{Res}_{t=c} \frac{t^{1 / 2}-t^{-1 / 2}}{t} D^{2} \varphi(t)=\operatorname{Res}_{t=c} \frac{t^{1 / 2}-t^{-1 / 2}}{t} \varphi(t) .
$$

Therefore, when we consider the residue of (21) of the form (22), we can replace $D^{2}$ with 1 , and, hence, we obtain (22).

\section{Proof of Theorem 1.1}

In this section, we prove Theorem 1.1, which states the topological invariance of the perturbative invariants of 3-manifolds with the first Betti number 1. We show "Kirby theorem" for surgery presentations of such 3-manifolds in Proposition 4.1, and prove Theorem 1.1 by introducing the $t$-through relation. To prove the theorem, we also show some properties of the $t$-through relation.

Let $M$ be a closed 3-manifold with the first Betti number 1. We choose a framed link $L^{\prime}$ in $S^{3}$ such that $M$ is obtained from $S^{3}$ by surgery along $L^{\prime}$. Then, $H^{1}(M ; \mathbb{Z})$ is presented by the kernel of the homomorphism $\mathbb{Z}^{n} \rightarrow \mathbb{Z}^{n}$ given by the multiplication of the linking matrix $A$ of $L^{\prime}$, and, hence, a cohomology class in $H^{1}(M ; \mathbb{Z})$ is presented by $\mathbf{a} \in \mathbb{Z}^{n}$ such that $A \mathbf{a}=0$. We can assume, without loss of generality, that all entries in the first row and the first column of $A$ are equal to 0 and $\mathbf{a}=\left(\begin{array}{llll}1 & 0 & \cdots & 0\end{array}\right)^{T}$, by changing $L^{\prime}$ by handle slide moves if necessary. This implies that the first component of $L^{\prime}$ has 0 framing and the other components are null-homologous in the complement of the first component. Further, we can assume that the first component of $L^{\prime}$ is the trivial knot $K_{0}$, because we can untie the first component by a sequence of crossing changes and each crossing change can be realized by a surgery along a small trivial knot winding around the crossing. It follows, by putting $L^{\prime}=K_{0} \cup L$, that, for a 3-manifold $M$ with the first Betti number 1, we can choose a framed link $K_{0} \cup L$ in $S^{3}$ as a surgery presentation of $M$ such that $K_{0}$ is the trivial knot with 0 framing and each component of $L$ is null-homologous in the complement of $K_{0}$.

Proposition 4.1 Let $K_{0}$ be the trivial knot with 0 framing in $S^{3}$, and let $L$ and $L^{\prime}$ be framed links in $S^{3}-K_{0}$ such that each component of them is null-homologous in $S^{3}-K_{0}$, and let $S_{K_{0} \cup L}^{3}$ and $S_{K_{0} \cup L^{\prime}}^{3}$ denote the 3-manifolds obtained from $S^{3}$ by surgery along $K_{0} \cup L$ and $K_{0} \cup L^{\prime}$ respectively. Then, $S_{K_{0} \cup L}^{3}$ and $S_{K_{0} \cup L^{\prime}}^{3}$ are homeomorphic if and only if $L$ and $L^{\prime}$ are related by a sequence of the KI and KII moves on the link and the KII moves over $K_{0}$, where the KI and KII moves are as shown in Figure 1. 


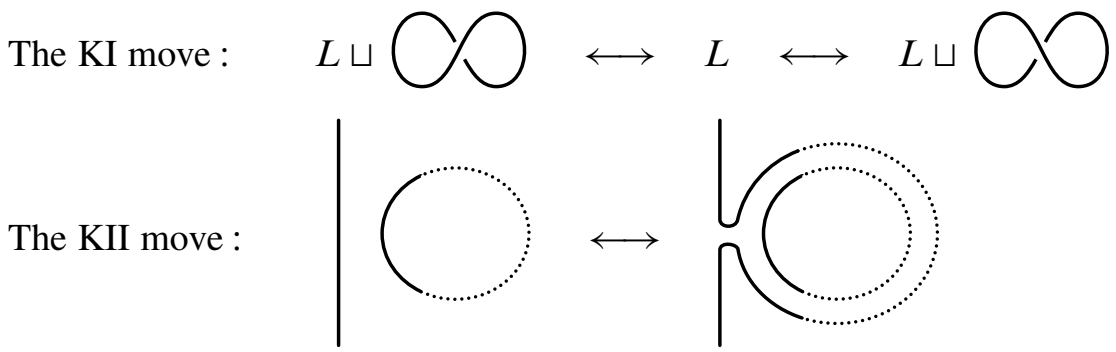

Figure 1: The KI and KII moves

Proof We denote by $L_{K_{0}}$ and $L_{K_{0}}^{\prime}$ the framed links in $S^{2} \times S^{1}$ obtained from $L$ and $L^{\prime}$ by surgery along $K_{0}$; note that they are null-homotopic in $S^{2} \times S^{1}$. Let $W_{L_{K_{0}}}$ and $W_{L_{K_{0}}^{\prime}}$ be the 4-manifolds obtained from $S^{2} \times S^{1} \times I$ by attaching 2-handles along $L_{K_{0}}$ and $L_{K_{0}}^{\prime}$ respectively. Then, $\partial W_{L_{K_{0}}} \cong \partial W_{L_{K_{0}}^{\prime}} \cong\left(S^{2} \times S^{1}\right) \sqcup \bar{M}$. Further, the diagram

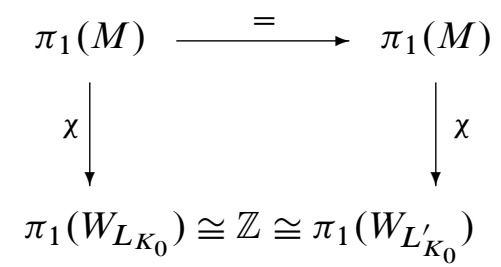

commutes, and $H_{4}(\mathbb{Z}) \cong H_{4}\left(S^{1}\right)=0$. Hence, by Fenn-Rourke [5, Theorem 6], $L_{K_{0}}$ and $L_{K_{0}}^{\prime}$ are related by the KI and KII moves. Therefore, $L$ and $L^{\prime}$ are related by the $\mathrm{KI}$ and KII moves on the link and the KII moves over $K_{0}$.

To show the invariance under the KII move over $K_{0}$, we introduce the $t$-through relation among Jacobi diagrams with labels of polynomials in $t$. We define the $t-$ through relation to be the relation generated by

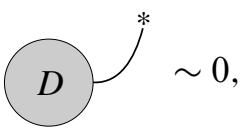

where $D$ is a Jacobi diagram with labels of polynomials in $t$, and we define a diagram with the marking to be the sum of diagrams obtained from the original diagram by connecting the marking at the right side of each $t$; for example,

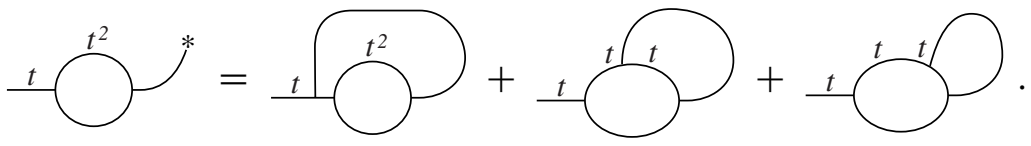


This operation is well-defined, since, for the equality,

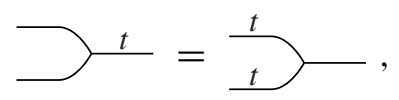

we have that

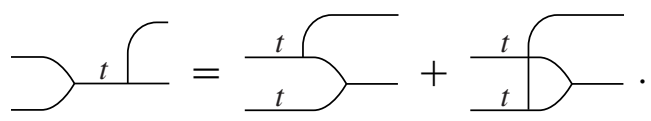

Lemma 4.2 Let $K_{0}$ be the trivial knot with 0 framing in $S^{3}$, and let $L$ and $L^{\prime}$ be framed links in $S^{3}-K_{0}$ such that each component of them is null-homologous in $S^{3}-K_{0}$. If $L$ and $L^{\prime}$ are related by the KII move over $K_{0}$, then $\chi^{-1} Z\left(K_{0} \cup L\right) \sqcup \Omega^{-1}$ and $\chi^{-1} Z\left(K_{0} \cup L^{\prime}\right) \sqcup \Omega^{-1}$ are related by the $t$-through relation.

Proof From the definition of the Kontsevich invariant (see, for example, the author's book [28]), we have that

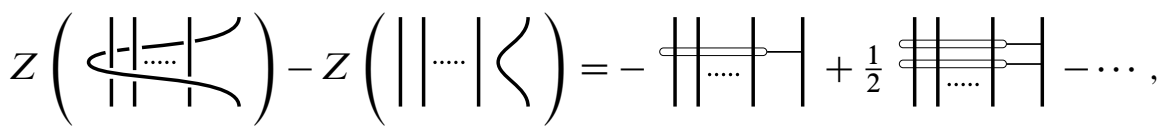

where

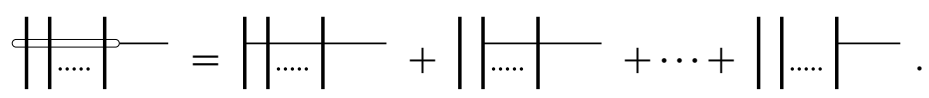

Further, from (16) and the definition of the $t$-through relation, we have that

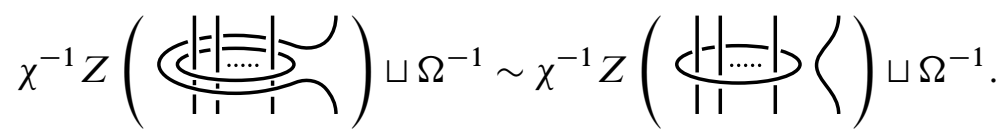

This implies the lemma.

Proof of Theorem 1.1 We recall the construction of the perturbative invariant $\tau(M ; c)$, where $M$ is a 3-manifold with the first Betti number 1 and $c$ is a zero of the Alexander polynomial $\Delta(t)$ of $M$. As mentioned before, we choose a framed link $K_{0} \cup L$ in $S^{3}$ as a surgery presentation of $M$ such that $K_{0}$ is the trivial knot with 0 framing and each component of $L$ is null-homologous in the complement of $K_{0}$. Let $N$ be the 3-manifold obtained from $S^{3}$ by surgery along $L$. By Proposition 3.1, the loop expansion of $W_{\mathfrak{s l}_{2}, V_{n}}\left(Z^{\mathrm{LMO}}(N, K)\right)$ is presented by (20) for some polynomials $P_{\ell}(t) \in \mathbb{Q}\left[t^{ \pm 1}\right]$. By using these $P_{\ell}(t)$, we defined the perturbative invariant by (3). Further, by Remark 3.3, we can replace $P_{\ell}(t)$ with $\widehat{P}_{\ell}(t)$, that is,

$$
\tau(M ; c)=-\frac{1}{2} \cdot \frac{1+c}{1-c} \sum_{\ell=0}^{\infty}\left(\operatorname{Res}_{t=c} \frac{\left(1-t^{-1}\right)^{2} \widehat{P}_{\ell}(t)}{\Delta(t)^{2 \ell+1}}\right)(q-1)^{\ell},
$$


where $\widehat{P}_{\ell}(t) \in \mathbb{Q}\left[t^{ \pm 1}\right]$ are determined by

$$
\begin{aligned}
& \sum_{\ell=0}^{\infty} \frac{\widehat{P}_{\ell}(\hat{t})}{\Delta(\hat{t})^{2 \ell+1}}(q-1)^{\ell} \underset{\left(\mathfrak{s l}_{2}\right)}{\equiv} \chi^{-1} Z^{\mathrm{LMO}}(N, K) \sqcup \Omega^{-1} \\
& =c_{+}^{-\sigma_{+}} c_{-}^{-\sigma_{-}} \int^{\mathrm{rat}} \chi^{-1} Z\left(K_{0} \cup L\right) d Y \sqcup \Omega^{-1} .
\end{aligned}
$$

By Proposition 4.1, it is sufficient to show the invariance of $\tau(M ; c)$ under the orientation change of $K_{0}$ and under the KI and KII moves on $L$ and under the KII moves over $K_{0}$. By the orientation change of $K_{0}$, labels of $\chi^{-1} Z\left(K_{0} \cup L\right)$ changes by replacing $t$ with $t^{-1}$. Since the $\mathfrak{s l}_{2}$ reduction is invariant under this change, we obtain the invariance of $\tau(M ; c)$ under the orientation change of $K_{0}$. The invariance of (23) under the KI and KII moves on $L$ is obtained from basic properties of the Aarhus integral; the invariance under the KI move is obtained since the change of the Aarhus integral under the KI move cancels with the change of the normalization factor $c_{+}^{-\sigma_{+}} c_{-}^{-\sigma_{-}}$, and the invariance under the KII move is obtained since the KII move can be presented by certain variable change of the Aarhus integral and the Aarhus integral is unchanged under such variable change; for details, see Bar-Natan, Garoufalidis, Rozansky and Thurston $[1 ; 2 ; 3]$ and Garoufalidis and Kricker [9]. Hence, it is sufficient to show the invariance of $\tau(M ; c)$ under the KII move over $K_{0}$.

When we change $K_{0} \cup L$ by the KII move over $K_{0}, \chi^{-1} Z\left(K_{0} \cup L\right) \sqcup \Omega^{-1}$ changes by the $t$-through relation by Lemma 4.2. Further, by Lemma 4.7, $\chi^{-1} Z^{\mathrm{LMO}}(N, K) \sqcup \Omega^{-1}$ changes by the $t$-through relation on Jacobi diagrams with labels of rational functions. Hence, the change of $\chi^{-1} Z^{\mathrm{LMO}}(N, K) \sqcup \Omega^{-1}$ is presented by

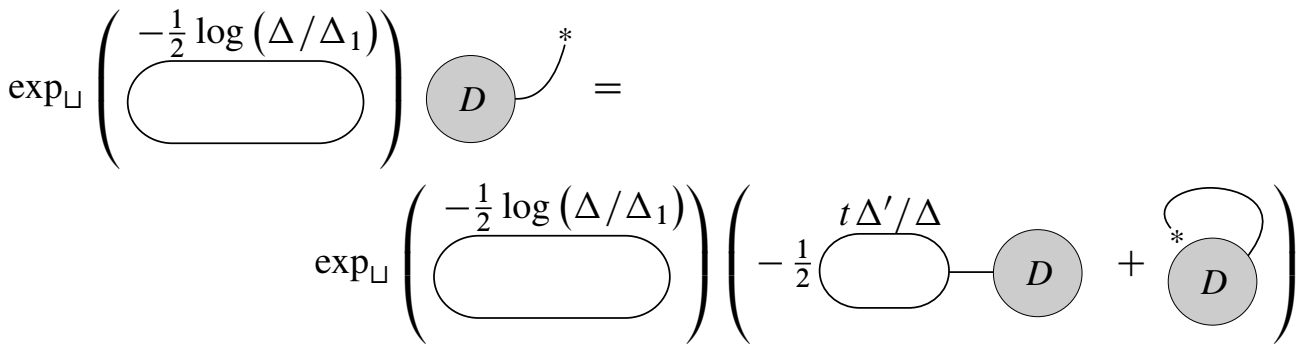

for some diagram $D$ with labels of rational functions, where we put $\Delta=\Delta(t)$, $\Delta_{1}=\Delta(1)$, and $\Delta^{\prime}=\frac{d}{d t} \Delta(t)$. By $\mathfrak{s l}_{2}$ reduction, it is sufficient to consider the case where

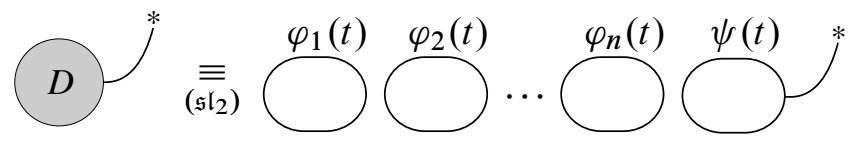


for some rational functions $\varphi_{i}(t)$ and $\psi(t)$ satisfying that $\varphi_{i}(t)=\varphi_{i}\left(t^{-1}\right), \varphi_{i}(1)=0$ and $\psi(t)=-\psi\left(t^{-1}\right)$. In this case,

$$
\begin{aligned}
& \bigcirc D=\underset{\left(\Delta^{\prime} \mathbb{L}_{2}\right)}{\equiv} 8 \frac{\hat{t} \Delta^{\prime}(\hat{t}) \psi(\hat{t})}{\Delta(\hat{t})} \prod_{i} 2 \varphi_{i}(\hat{t}), \\
& \overbrace{D} \underset{\left(\mathfrak{s l}_{2}\right)}{\equiv} 8 \hat{t} \varphi_{1}(\hat{t}) \psi(\hat{t}) \prod_{i \neq 1} 2 \varphi_{i}(\hat{t})+8 \hat{t} \varphi_{2}(\hat{t}) \psi(\hat{t}) \prod_{i \neq 2} 2 \varphi_{i}(\hat{t})+\cdots \\
& +8 \hat{t} \varphi_{n}(\hat{t}) \psi(\hat{t}) \prod_{i \neq n} 2 \varphi_{i}(\hat{t})+4\left(\hat{t} \psi^{\prime}(\hat{t})+\frac{\hat{t}+1}{\hat{t}-1} \psi(\hat{t})\right) \prod_{i} 2 \varphi_{i}(\hat{t}) \\
& =4 \hat{t}\left(\psi(\hat{t}) \prod_{i} 2 \varphi_{i}(\hat{t})\right)^{\prime}+4 \frac{\hat{t}+1}{\hat{t}-1} \psi(\hat{t}) \prod_{i} 2 \varphi_{i}(\hat{t}),
\end{aligned}
$$

Hence, by putting $\varphi(t)=4 \psi(t) \prod_{i} 2 \varphi_{i}(t)$,

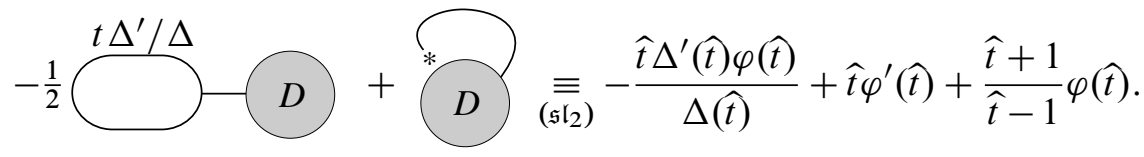

Therefore, the change of $\sum_{\ell}\left(\widehat{P}_{\ell}(t) / \Delta(t)^{2 \ell+1}\right)(q-1)^{\ell}$ is given by

$$
\frac{\Delta(1)}{\Delta(t)}\left(-\frac{t \Delta^{\prime}(t) \varphi(t)}{\Delta(t)}+t \varphi^{\prime}(t)+\frac{t+1}{t-1} \varphi(t)\right)=\Delta(1)\left(t\left(\frac{\varphi(t)}{\Delta(t)}\right)^{\prime}+\frac{t+1}{t-1} \cdot \frac{\varphi(t)}{\Delta(t)}\right) .
$$

Further, the change of the defining formula of $\tau(M ; c)$ is given by

$$
\begin{aligned}
-\frac{1}{2} \cdot \frac{1+c}{1-c} \operatorname{Res}\left(1-t^{-1}\right)^{2} \Delta(1)\left(t\left(\frac{\varphi(t)}{\Delta(t)}\right)^{\prime}+\frac{t+1}{t-1} \cdot \frac{\varphi(t)}{\Delta(t)}\right) & \\
= & -\frac{\Delta(1)}{2} \cdot \frac{1+c}{1-c} \operatorname{Res}\left(\frac{\left(t+t^{-1}-2\right) \varphi(t)}{\Delta(t)}\right)^{\prime}=0,
\end{aligned}
$$

since the residue of the differential of some function is always equal to 0 . Hence, we obtain the invariance of $\tau(M ; c)$ under the KII move over $K_{0}$.

In order to show Lemma 4.7 below, we extend the definition of the $t$-through relation to Jacobi diagrams with labels of rational functions. To extend it, we show some properties of the marking connected to a label of a rational function, as follows. We 
define the marking connected to a label of $t^{k}$ by

$$
\begin{aligned}
& \overbrace{{ }^{*}{ }^{k}}^{t_{t^{k}}(\overbrace{t^{k-1}(t}}+\cdots+\overbrace{t \int t^{k-1}} \\
& =\overbrace{t^{k} \overbrace{}^{1+t+\cdots+t^{k-1}}}^{k}=\overbrace{}^{k{ }^{\left(t^{k}-1\right) /(t-1)}}
\end{aligned}
$$

for $k \in \mathbb{Z}$. By extending this definition linearly, we define the marking connected to a label of a polynomial $f \in \mathbb{Q}\left[t^{ \pm 1}\right]$. Then, we can show, for polynomials $f, g \in \mathbb{Q}\left[t^{ \pm 1}\right]$, that

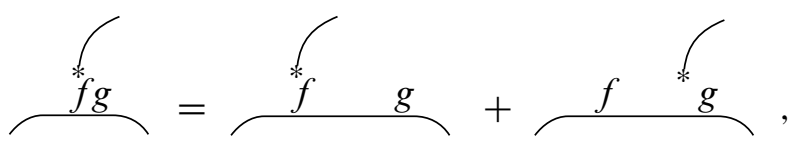

by reducing the proof to the case where $f=t^{i}$ and $g=t^{j}$. Further, we define the marking connected to a label of a rational function $f / g$ (for $f, g \in \mathbb{Q}\left[t^{ \pm 1}\right]$ with $g(1) \neq 0)$ by

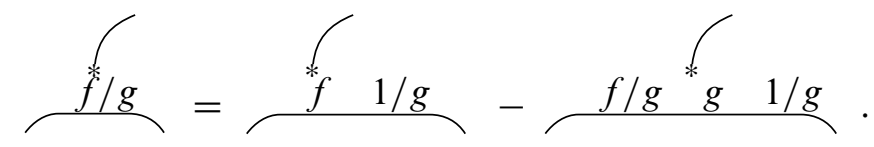

In particular,

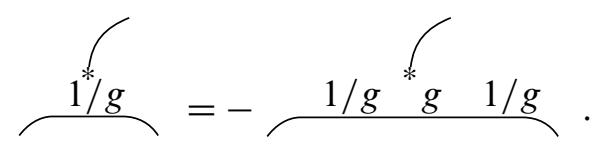

Note that these two formulas imply that

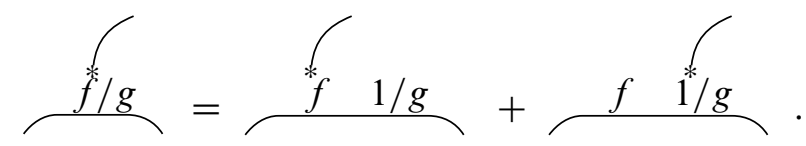

Lemma 4.3 For polynomials $f, g \in \mathbb{Q}\left[t^{ \pm 1}\right]$ with $g(1) \neq 0$,

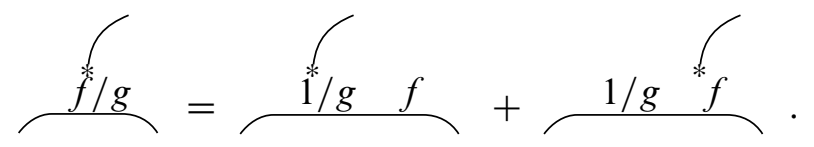

Proof By definition, it is sufficient to show that

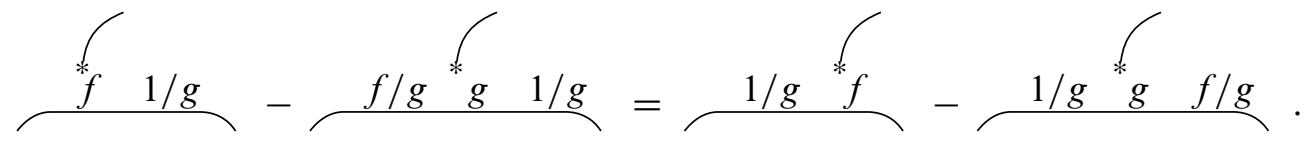

By multiplying $g$ from both sides of each term, this formula is equivalent to the formula

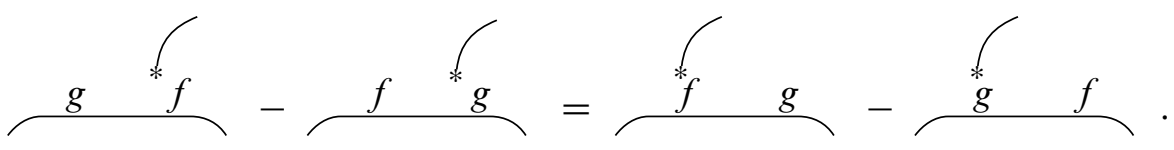


This is obtained by applying (25) to diagrams with labels of $f g$ and $g f$.

Lemma 4.4 For polynomials $f, g \in \mathbb{Q}\left[t^{ \pm 1}\right]$ with $f(1), g(1) \neq 0$,

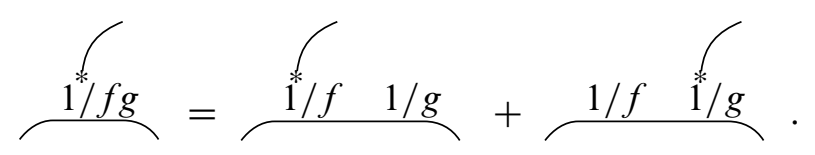

Proof By definition, it is sufficient to show that

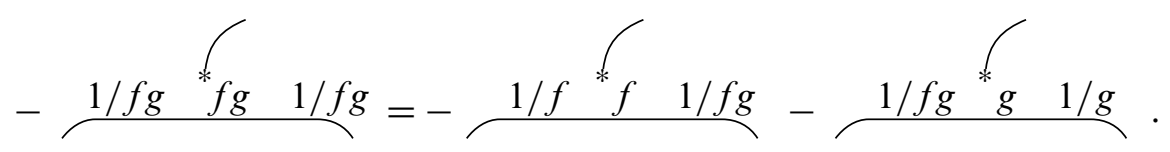

This is obtained by applying (25) to the label of $f g$.

Lemma 4.5 For rational functions $\varphi$, $\psi$ of $t$,

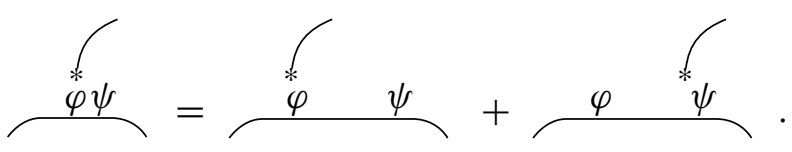

Proof We put $\varphi=f_{1} / g_{1}$ and $\psi=f_{2} / g_{2}$ for polynomials $f_{i}, g_{i} \in \mathbb{Q}\left[t^{ \pm 1}\right]$ with $g_{i}(1) \neq 0$. Then, the left-hand side of the required formula is equal to

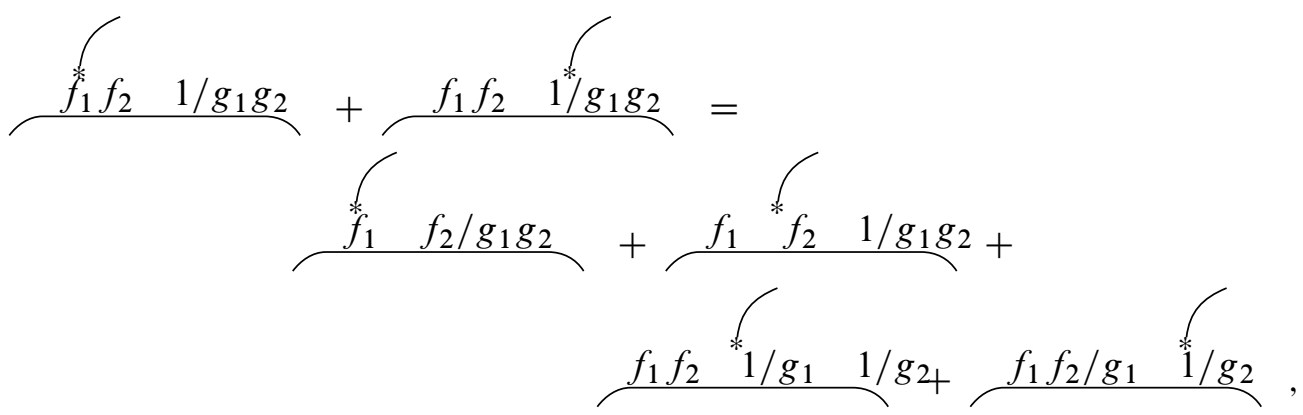

where the equality is obtained from (25) and Lemma 4.4. Further, since we can exchange the order of $f_{2}$ and $1 / g_{1}$ in the second and third terms by (26) and Lemma 4.3, the above formula is equal to

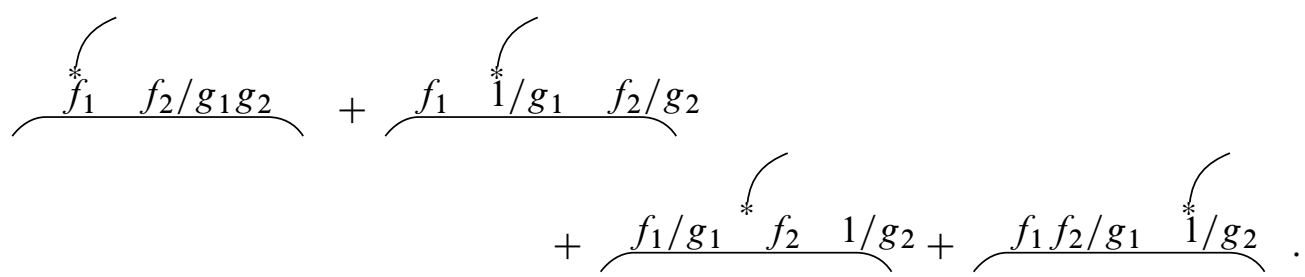

By definition, the right-hand side of the required formula is equal to this formula. 
Extending the previous definition, we redefine the $t$-through relation to be the relation generated by

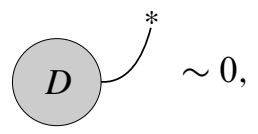

where $D$ is a Jacobi diagram with labels of rational functions of $t$, and we define a diagram with the marking to be the sum of diagrams obtained from the original diagram by connecting the marking at each label; for example,

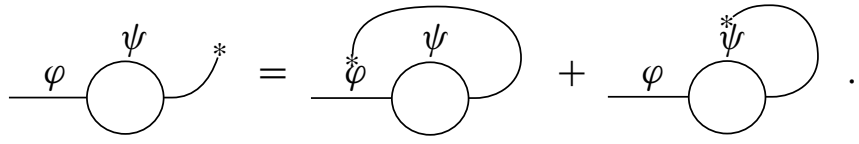

Lemma 4.6 For a rational function $\varphi$ of $t$,

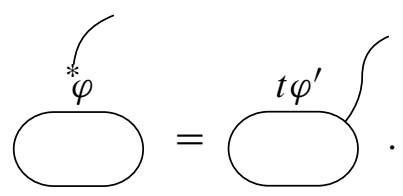

Proof When $\varphi=t^{k}$, we obtain the lemma,

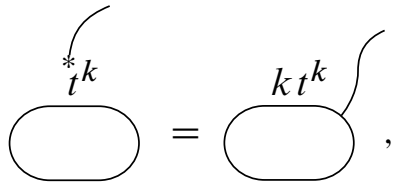

since this equality is obtained from (24) and the property

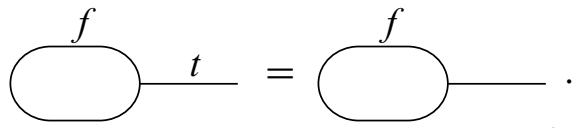

In general, putting $\varphi=f / g$ for polynomials $f$ and $g$ in $t^{ \pm 1}$, we obtain the lemma,

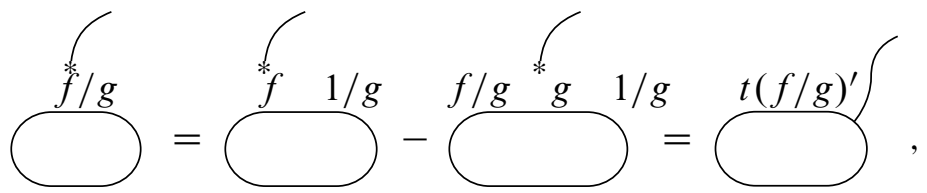

where we obtain the second equality in the same way as above.

Extending Lemma 4.6, we define the marking connected to a label of $\log f$ on a loop for a polynomial $f$ in $t^{ \pm 1}$ by

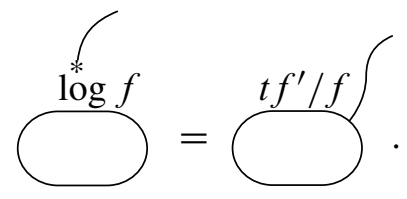


Lemma 4.7 If $\chi^{-1} Z\left(K_{0} \cup L\right)$ and $\chi^{-1} Z\left(K_{0} \cup L^{\prime}\right)$ are related by the $t$-through relation, then $\int^{\text {rat }} \chi^{-1} Z\left(K_{0} \cup L\right) d Y$ and $\int^{\text {rat }} \chi^{-1} Z\left(K_{0} \cup L^{\prime}\right) d Y$ are related by the $t$-through relation on Jacobi diagrams with labels of rational functions.

Proof When $L$ is of 1 component, we show the lemma, as follows. In this case, the difference of the $t$-through relation is generated by the form

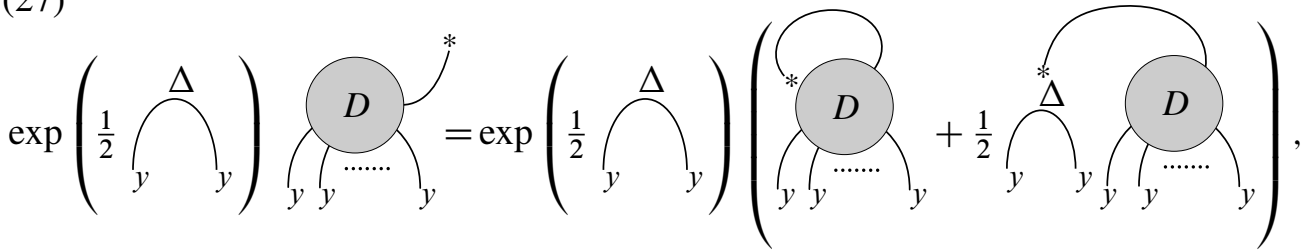

where $D$ is a diagram with $2 k$ legs. By the rational version of the Aarhus integral, this is taken to

(28)

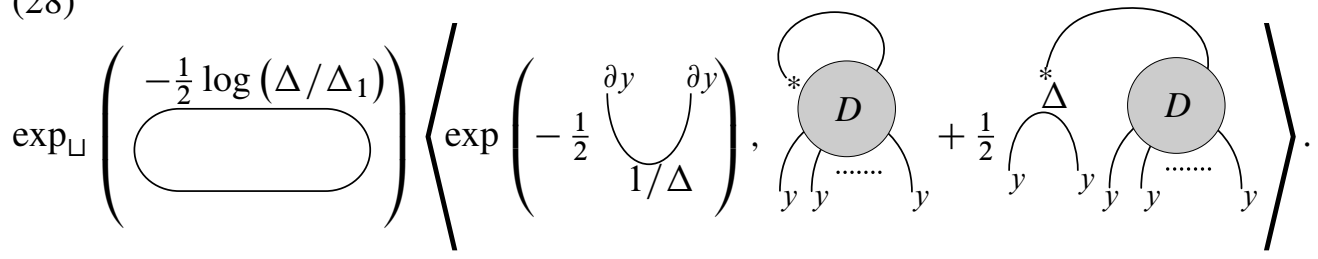

Further, the part of the bracket is calculated as
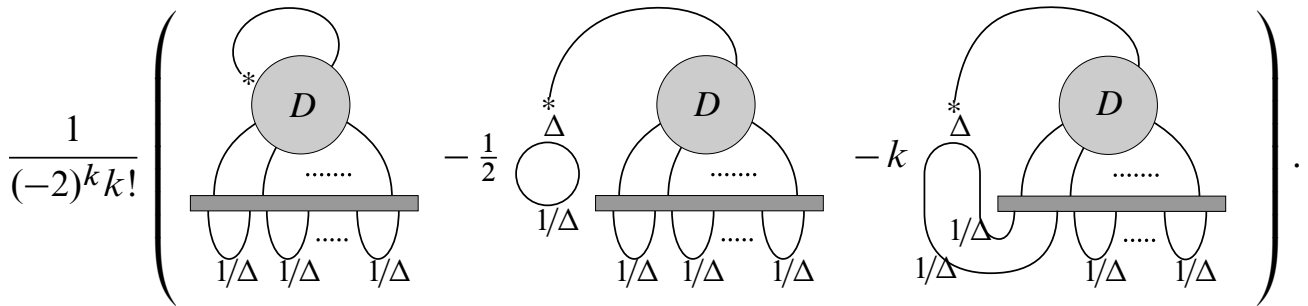

Hence, (28) is equal to

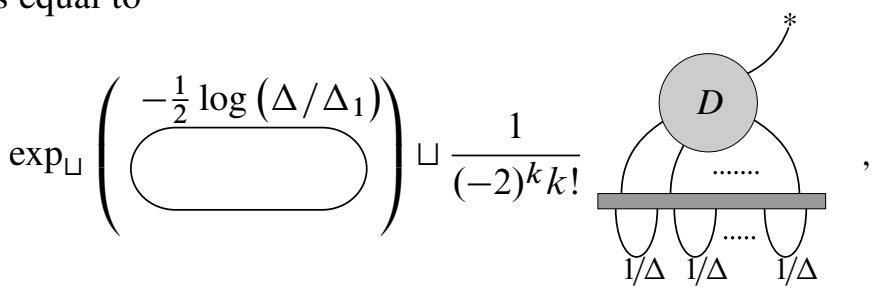

where we use

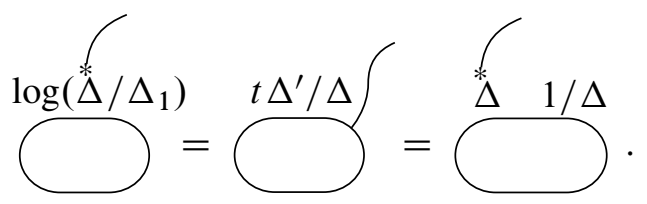


Therefore, the Aarhus integral of (27) is presented by the form (29), and this implies the lemma. Note that (29) can also be presented by

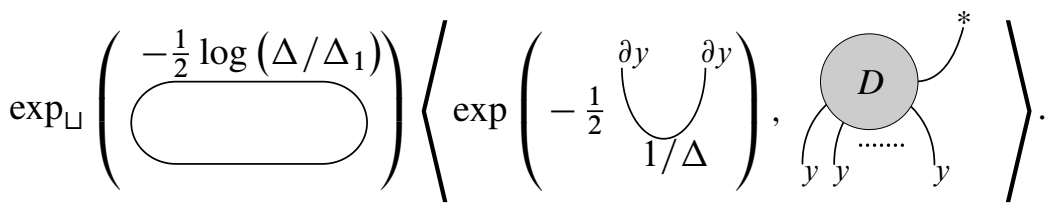

In general, the difference of the $t$-through relation is generated by the form,

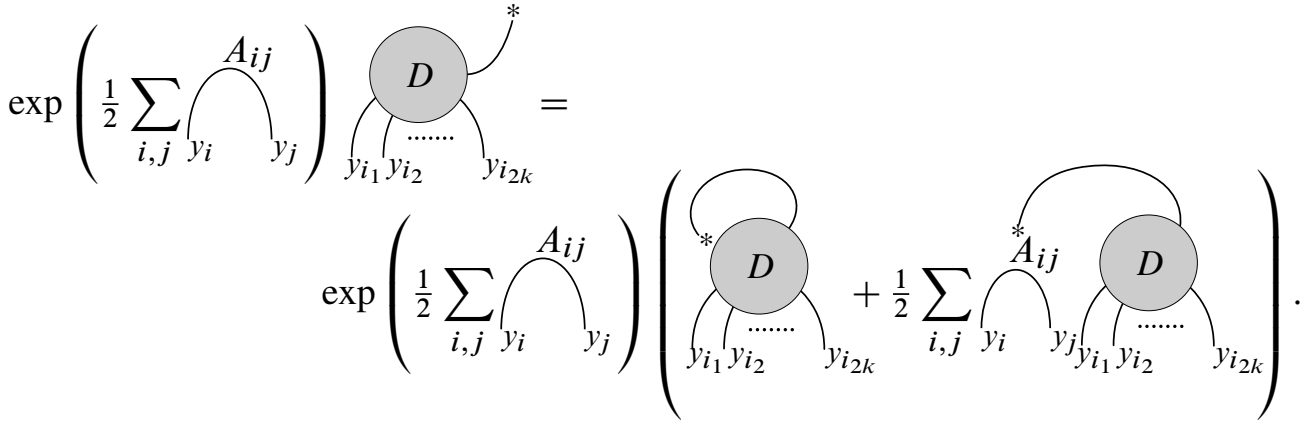

Similarly as the above case, we can show, by replacing $\Delta$ and $1 / \Delta$ with $A_{i j}$ and $\left(A^{-1}\right)_{i j}$, that the Aarhus integral takes the above formula to

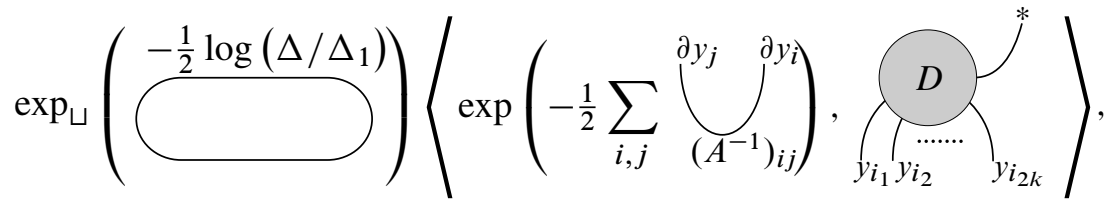

where, instead of the second equality of (30), we use

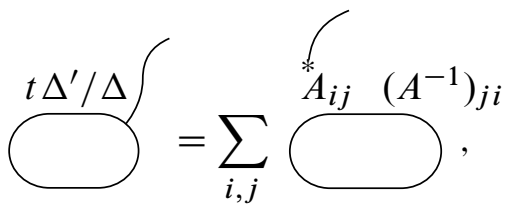

since $\Delta^{\prime} / \Delta=\operatorname{trace}\left(A^{\prime} \cdot A^{-1}\right)=\sum_{i, j} A_{i j}^{\prime} \cdot\left(A^{-1}\right)_{j i}$. This completes the proof.

Lemma 4.8 Let $D_{1}$ and $D_{2}$ be Jacobi diagrams with a marking such that they are related by the procedure of $\mathfrak{s l}_{2}$ reduction explained in Section 3.2. Then, the diagrams obtained from $D_{1}$ and $D_{2}$ by applying the marking on themselves are related by $\mathfrak{s l}_{2}$ reduction. 
The lemma implies that the operation of the marking $*$ commutes with $\mathfrak{s l}_{2}$ reduction. In other words, it implies that if

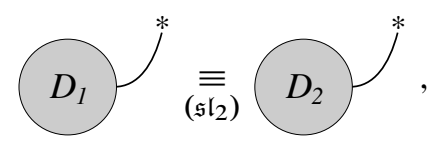

then

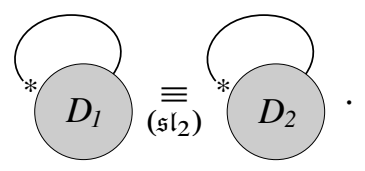

Proof The procedure of $\mathfrak{s l}_{2}$ reduction mainly consists of the application of (17), and this commutes with the operation of the marking $*$. The non-trivial part is the procedure of ignoring a trivalent vertex whose adjacent edges have labels $\varphi_{i}(t)(i=1,2,3)$ with $\varphi_{i}(1)=0$. Hence, it is sufficient to show that
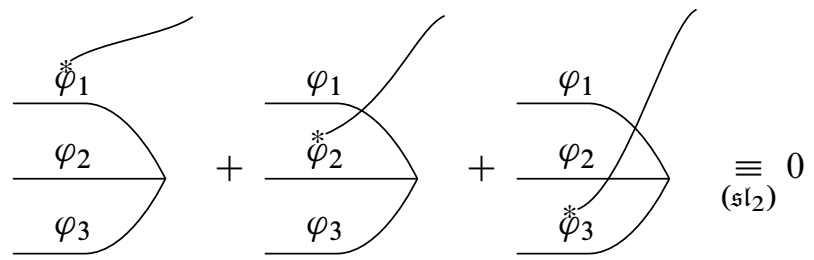

for rational functions $\varphi_{i}(t)$ with $\varphi_{i}(1)=0$. We put $\varphi_{i}=f_{i} / g_{i}$ for polynomials $f_{i}$ and $g_{i}$ with $f_{i}(1)=0$. Then, the left-hand side of the above formula is equal to

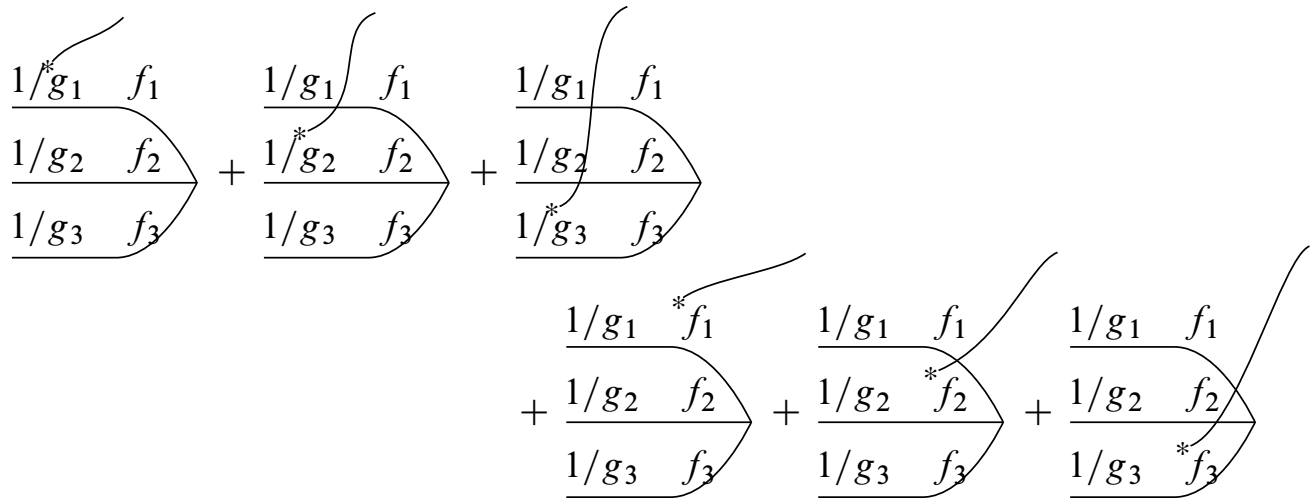

Further, the sum of the first three terms is equivalent to 0 by $\mathfrak{s l}_{2}$ reduction, since $f_{i}(1)=0$ for all $i$. Hence, it is sufficient to show (31) when $\varphi_{i}=f_{i}$. Further, the proof can be reduced to the case where $f_{i}=t^{k_{i}}-1$. In this case, we can check (31) concretely by (24) and (17). 
Lemma 4.9 For a rational function $\varphi(t)$ with $\varphi(t)=-\varphi\left(t^{-1}\right)$,

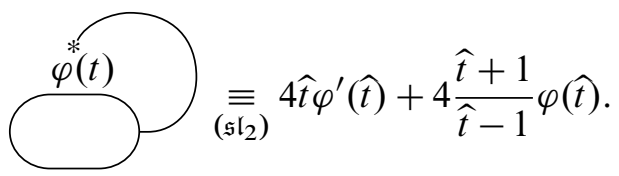

Proof We put $\varphi(t)=f(t) / g(t)$ for polynomials $f(t)$ and $g(t)$ with $f(t)=-f\left(t^{-1}\right)$ and $g(t)=g\left(t^{-1}\right)$. By definition,

Further,
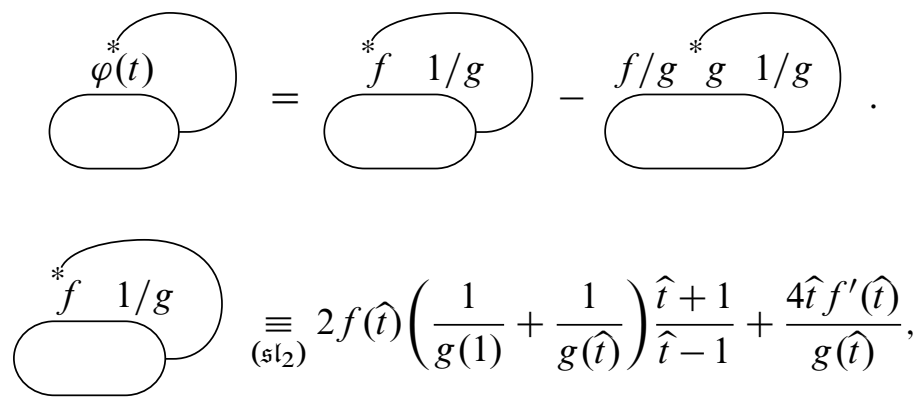

where we can show this equivalence by (18) and (24) putting $f(t)=t^{k}+t^{-k}$. In a similar way, we can show that

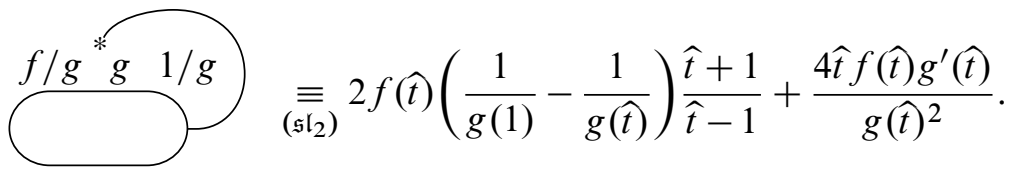

Hence,

$$
\overbrace{}^{*}(t) \underset{\left(\mathfrak{s l _ { 2 } )}\right.}{\equiv} 4 \hat{t}\left(\frac{f^{\prime}(\hat{t})}{g(\hat{t})}-\frac{f(\hat{t}) g^{\prime}(\hat{t})}{g(\hat{t})^{2}}\right)+4 \frac{\hat{t}+1}{\hat{t}-1} \cdot \frac{f(\hat{t})}{g(\hat{t})},
$$

which implies the required formula.

\section{Properties of the perturbative invariants}

In this section, we show some properties of the perturbative invariants. As before, we let $M$ be a 3-manifold with the first Betti number 1 .

Proposition 5.1 For a zero $c$ of $\Delta(t), \tau(M ; c)=\tau\left(M ; c^{-1}\right)$.

Proof It is sufficient to show that $\lambda_{\ell}(M ; c)=\lambda_{\ell}\left(M ; c^{-1}\right)$. By definition, $\lambda_{\ell}(M ; c)$ is presented by the form,

$$
\frac{1+c}{1-c} \operatorname{Res} \frac{\varphi(t)}{t}=\frac{1+c}{1-c} \cdot \frac{1}{2 \pi \sqrt{-1}} \int_{\gamma(c)} \varphi(t) \frac{d t}{t}
$$


for some rational function $\varphi(t)$ with $\varphi(t)=\varphi\left(t^{-1}\right)$, where $\gamma(c)$ is a small loop winding around $c$. Further, $\lambda_{\ell}\left(M ; c^{-1}\right)$ is calculated by the same formula replacing the variable by $t=1 / u$. Since $d t / t=-d u / u$ and $(1+c) /(1-c)=-\left(1+c^{-1}\right) /\left(1-c^{-1}\right)$, we obtain that $\lambda_{\ell}(M ; c)=\lambda_{\ell}\left(M ; c^{-1}\right)$.

Proposition 5.2 For a rational homology 3-sphere $N$,

$$
\tau(M \# N ; c)=\tau(M ; c) \tau(N)\left|H_{1}(N ; \mathbb{Z})\right|,
$$

where $M \# N$ denotes the connected sum of $M$ and $N, c$ is 0 or a zero of the Alexander polynomial of $M$, and $\tau(N) \in \mathbb{Q} \llbracket q-1 \rrbracket$ denotes the perturbative $S O(3)$ invariant of $N$ (see the author's paper [26]).

Proof By the connected sum of $N$, the perturbative invariant $\tau(M ; c)$ changes by the multiple of

$$
W_{\mathfrak{s l}_{2}}\left(Z^{\mathrm{LMO}}(N)\right)=\left|H_{1}(N ; \mathbb{Z})\right| \tau(N) \in \mathbb{Q} \llbracket q-1 \rrbracket,
$$

where the equality is derived from the universality of the LMO invariant among perturbative invariants of rational homology 3-spheres, see the author's paper [27]. Hence, we obtain the proposition.

\section{Proposition 5.3}

$$
\lambda_{0}(M ; 0)= \begin{cases}1 & \text { if } \operatorname{deg} \Delta(t)=0, \\ -\frac{\Delta(1)}{\Delta^{\prime \prime}(1)} & \text { if } \operatorname{deg} \Delta(t)=1, \\ 0 & \text { if } \operatorname{deg} \Delta(t)>1 .\end{cases}
$$

For a zero $c$ of $\Delta(t)$ of multiplicity $m \leq 2$,

$$
\lambda_{0}(M ; c)= \begin{cases}\frac{\left(1-c^{-2}\right) \Delta(1)}{2 \Delta^{\prime}(c)} & \text { if } m=1, \\ \left(\frac{2(c+1)}{c^{3}}+\frac{\left(c^{-2}-1\right) \Delta^{\prime \prime \prime}(c)}{3 \Delta^{\prime \prime}(c)}\right) \frac{\Delta(1)}{\Delta^{\prime \prime}(c)} & \text { if } m=2 .\end{cases}
$$

Proof We calculate $\lambda_{0}(M ; 0)$, as follows. By definition,

$$
\lambda_{0}(M ; 0)=-\frac{\Delta(1)}{2} \operatorname{Res} \frac{t+t^{-1}-2}{t \Delta(t)} .
$$

If $\operatorname{deg} \Delta(t) \leq 1$, putting $\Delta(t)=b_{0}$ or $\Delta(t)=b_{1}\left(t+t^{-1}\right)+b_{0}$, the required formula can be shown concretely. If $\operatorname{deg} \Delta(t)>1$, putting $\Delta(t)=b_{n}\left(t^{n}+t^{-n}\right)+\cdots$, the function of the residue has the form $\left(t^{n}-2 t^{n-1}+t^{n-2}\right) /\left(b_{n} t^{2 n}+\cdots+b_{n}\right)$, and it has no pole at $t=0$. Hence, its residue equals 0 . 
We calculate $\lambda_{0}(M ; c)$, as follows. By definition,

$$
\lambda_{0}(M ; c)=-\frac{\Delta(1)}{2} \cdot \frac{1+c}{1-c} \operatorname{Res} \frac{t+t^{-1}-2}{t \Delta(t)} .
$$

If $c$ is a zero of $\Delta(t)$ of multiplicity 1 , the residue is calculated as

$$
\operatorname{Res}_{t=c} \frac{t+t^{-1}-2}{t \Delta(t)}=\lim _{t \rightarrow c} \frac{(t-c)\left(t+t^{-1}-2\right)}{t \Delta(t)}=\lim _{t \rightarrow c} \frac{t+t^{-1}-2}{t \Delta^{\prime}(t)}=\frac{(c-1)^{2}}{c^{2} \Delta^{\prime}(c)},
$$

and we obtain the required formula. If $c$ is a zero of $\Delta(t)$ of multiplicity 2, putting $\Delta(t)=(t-c)^{2} f(t)$, the residue is calculated as

$$
\begin{aligned}
\operatorname{Res}_{t=c} \frac{t+t^{-1}-2}{t \Delta(t)} & =\operatorname{Res}_{t=c}\left(\frac{t+t^{-1}-2}{t(t-c)^{2} f(t)}-\frac{c+c^{-1}-2}{c(t-c)^{2} f(c)}\right) \\
& =\lim _{t \rightarrow c} \frac{1}{t-c}\left(\frac{t+t^{-1}-2}{t f(t)}-\frac{c+c^{-1}-2}{c f(c)}\right) \\
& =\left.\frac{d}{d t}\left(\frac{t+t^{-1}-2}{t f(t)}\right)\right|_{t=c} .
\end{aligned}
$$

Hence, noting that $\Delta^{\prime \prime}(c)=2 f(c)$ and $\Delta^{\prime \prime \prime}(c)=6 f^{\prime}(c)$, we obtain the required formula.

\subsection{Clasper surgery formula}

In this section, we show a surgery formula of the perturbative invariants under clasper surgery; for claspers, see Habiro [12]. It follows from this surgery formula that the coefficients of the perturbative invariants are independent invariants.

Let $F$ be a Seifert surface of a knot $K$ in an integral homology 3-sphere. The Seifert form $H_{1}(F) \otimes H_{1}(F) \rightarrow \mathbb{R}$ is defined by taking $a \otimes b$ to the linking number of $a$ and $b^{+}$, where $b^{+}$denotes the puss-off of $b$ in the normal direction of $F$. The Seifert form is presented by a Seifert matrix $V$, fixing a basis of $H_{1}(F)$. We denote by $e_{x}, e_{y}$ the vectors presenting cohomology classes $x, y \in H^{1}(F)$ for the basis. The scaler $e_{x}^{T}\left(t^{1 / 2} V-t^{-1 / 2} V^{T}\right)^{-1} e_{y}$ depends only on the Seifert form and $x, y \in H^{1}(F)$, independently of the choice of a basis of $H_{1}(F)$. The Alexander polynomial of the knot is given by $\Delta(t)=\operatorname{det}\left(t^{1 / 2} V-t^{-1 / 2} V^{T}\right)$. A leaf of a clasper in the complement of a Seifert surface $F$ of a knot is associated with a cohomology class in $H^{1}(F)$ counting cycles as

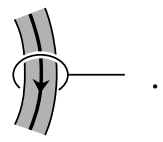


Let $K$ be a knot with 0 framing in an integral homology 3 -sphere, and let $M$ be the 3-manifold obtained from the integral homology 3-sphere by surgery along $K$. Consider a graph clasper $C$ of the following form, embedded in the complement of a Seifert surface $F$ of a knot $K$. Let $x, y$ be cohomology classes in $H^{1}(F)$ associated with the leaves of the graph clasper.

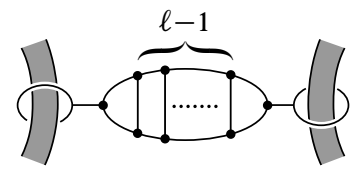

Proposition 5.4 Let $M$ and $C$ be as above, and let $M_{C}$ denote the 3-manifold obtained from $M$ by surgery along $C$. Then, the change of the perturbative invariants by the clasper surgery is presented by

$$
\begin{aligned}
\lambda_{\ell}\left(M_{C} ; c\right)-\lambda_{\ell}(M ; c) & =-\frac{1}{2} \cdot \frac{1+c}{1-c} \operatorname{Res} \frac{2^{\ell+2}\left(t^{1 / 2}-t^{-1 / 2}\right)^{3}}{t \Delta(t)} e_{x}^{T}\left(t^{1 / 2} V-t^{-1 / 2} V^{T}\right)^{-1} e_{y}, \\
\lambda_{k}\left(M_{C} ; c\right) & =\lambda_{k}(M ; c)
\end{aligned}
$$

for any $k<\ell$.

Proof In the same way as [33, Proposition 4.17], we have that

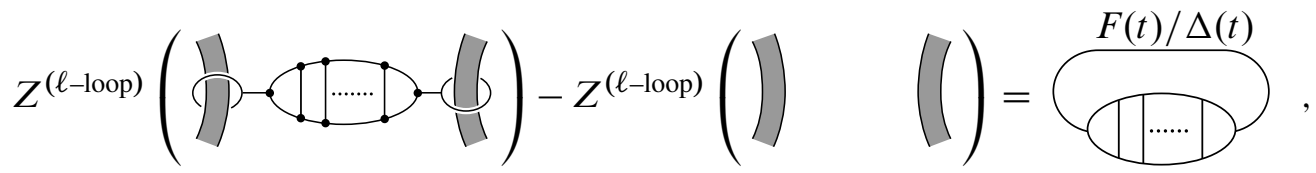

where $Z^{(\ell-\text { loop })}(K)$ denotes the $\ell$-loop part of $\log \chi^{-1} Z(K)$, and

$$
\frac{F(t)}{\Delta(t)}=-\left(t^{1 / 2}-t^{-1 / 2}\right) e_{x}^{T}\left(t^{1 / 2} V-t^{-1 / 2} V^{T}\right)^{-1} e_{y}
$$

Hence, by this clasper surgery, $P_{\ell}(t) / \Delta(t)^{2 \ell+1}$ changes by

$$
\frac{2^{\ell+2}\left(t^{1 / 2}-t^{-1 / 2}\right)}{\Delta(t)} e_{x}^{T}\left(t^{1 / 2} V-t^{-1 / 2} V^{T}\right)^{-1} e_{y},
$$

keeping $P_{k}(t)$ unchanged for any $k<\ell$. Therefore, from the definition of the perturbative invariants, we obtain the proposition.

We can obtain similar surgery formulas for other forms of the graph clasper, in the same way as in the author's paper [33]. 


\subsection{Stability of the perturbative invariants for finite cyclic covers}

There is a natural $d$-fold cyclic cover $M_{d}$ of a 3-manifold $M$ with $b_{1}(M)=1$ (defined below). By regarding the perturbative invariant of $M_{d}$ as an invariant of $M$ (assuming that $b_{1}\left(M_{d}\right)=1$ ), we have an infinite series of invariants of $M$. In this section, we show that this series is stable for sufficiently large $d$ in the cases of Propositions 5.5 and 5.6.

For a positive integer $d$, let $M_{d}$ be the $d$-fold cyclic cover of $M$ induced by the homomorphism

$$
\pi_{1}(M) \longrightarrow H_{1}(M ; \mathbb{Z}) \longrightarrow \mathbb{Z} \longrightarrow \mathbb{Z} / d \mathbb{Z},
$$

where the first map is the abelianization, the second map is the map ingoring the torsion part, and the third map is the projection. We assume that $b_{1}\left(M_{d}\right)=1$, that is, the Alexander polynomial $\Delta(t)$ of $M$ does not have a zero of a $d$ th root of unity. We choose a rational homology 3-sphere $N$ and a null-homologous knot $K$ in $N$ such that $M$ is obtained from $N$ by 0 surgery along $K$. Extending the above homomorphism, we consider the homomorphism,

$$
\pi_{1}(N-K) \longrightarrow \pi_{1}(M) \longrightarrow H_{1}(M ; \mathbb{Z}) \longrightarrow \mathbb{Z} \longrightarrow \mathbb{Z} / d \mathbb{Z},
$$

and this homomorphism induces the $d$-fold cyclic cover $\widetilde{N-K}$ of $N-K$ as a subset of $M_{d}$. The above homomorphism is equal to

$$
\pi_{1}(N-K) \longrightarrow H_{1}(N-K ; \mathbb{Z}) \cong H_{1}(N ; \mathbb{Z}) \oplus \mathbb{Z} \longrightarrow \mathbb{Z} \longrightarrow \mathbb{Z} / d \mathbb{Z},
$$

where the second map is the projection from $\mathbb{Z}$ ignoring $H_{1}(N ; \mathbb{Z})$. Let $N_{d}$ be the $d$-fold cyclic cover of $N$ branched along $K$ induced by the above homomorphism, and let $K_{d}$ be the knot of the branch set in $N_{d}$. Since $N_{d}-K_{d}$ is the $d$-fold cyclic cover of $N-K$ induced by the above homomorphism, $N_{d}-K_{d}$ is equal to $\widehat{N-K}$. Hence, $M_{d}$ is obtained from $N_{d}$ by 0 surgery along $K_{d}$. Further, the Alexander polynomial of $K_{d}$ is presented by

$$
\Delta_{K_{d}}(t)= \pm \prod_{\zeta^{d}=1} \Delta\left(\zeta t^{1 / d}\right),
$$

where we choose the sign so that $\Delta_{K_{d}}(1)>0$. Note that, for a zero $c$ of $\Delta(t), c^{d}$ is a zero of $\Delta_{K_{d}}(t)$.

We define the total signature of a 3-manifold $M$ with $b_{1}(M)=1$, as follows. As in Section $4, M$ is obtained from $S^{3}$ by surgery along some framed link $K_{0} \cup L$ such that $K_{0}$ is the trivial knot and each component of $L$ is null-homologous in $S^{3}-K_{0}$. Let $\widetilde{L}$ be the preimage of $L$ in the $d$-fold cyclic cover of $S^{3}$ branched along $K_{0}$, and 
let $A$ and $A_{d}$ be the linking matrices of $L$ and $\tilde{L}$ respectively. We define the total $d$-signature $\sigma_{d}$ of $M$ by

$$
\sigma_{d}(M)=\sigma\left(A_{d}\right)-d \sigma(A),
$$

where $\sigma(\cdot)$ denotes the signature of a symmetric matrix. We can verify, by Proposition 4.1, that this is an invariant of $M$.

Proposition 5.5 When $\Delta(t)$ is of degree 0 , for any positive integer $\ell$, the degree $\leq \ell$ part of $\left(q^{-(3 / 4) \sigma_{d}(M)} \tau\left(M_{d} ; 0\right)\right)^{1 / d}$ is stable (that is, constant) for sufficiently large $d$.

Hence, the stable part is presented by

$$
\tilde{\tau}(M ; 0)=\lim _{d \rightarrow \infty}\left(q^{-(3 / 4) \sigma_{d}(M)} \tau\left(M_{d} ; 0\right)\right)^{1 / d} \in \mathbb{Q} \llbracket q-1 \rrbracket .
$$

Proof of Proposition 5.5 When $\Delta(t)$ is of degree 0 , the loop expansion (see Section 3.1) of $Z^{\mathrm{LMO}}(N, K)$ is presented by

$$
\begin{aligned}
& \chi^{-1} Z^{\mathrm{LMO}}(N, K)= \\
& Z^{\mathrm{LMO}}(N) \sqcup \Omega \sqcup \exp _{\sqcup}\left(\begin{array}{l}
\text { linear sum of connected Jacobi diagrams } \\
\text { with labels of polynomials in } t^{ \pm 1}
\end{array}\right) .
\end{aligned}
$$

Its $d$-fold cover is calculated in the way shown by Garoufalidis and Kricker [8]; in particular, in this case,

$\chi^{-1} Z^{\mathrm{LMO}}\left(N_{d}, K_{d}\right) \sim$

$$
e^{\sigma_{d}(M) \theta / 16} \sqcup Z^{\mathrm{LMO}}(N)^{d} \sqcup \Omega \sqcup \exp _{\sqcup}\left(d \cdot\left(\begin{array}{l}
\text { constant terms of } \\
\text { the above linear sum }
\end{array}\right)\right),
$$

where $\theta$ is the Jacobi diagram of the $\theta$ graph, and this formula means that, for any positive integer $\ell$, the $(\leq \ell)$-loop parts of both sides are equal for sufficiently large $d$. Hence, the $\mathfrak{s l}_{2}$ reduction of $\chi^{-1} Z^{\mathrm{LMO}}\left(N_{d}, K_{d}\right) \sqcup \Omega^{-1}$ is stably presented by

$$
q^{(3 / 4) \sigma_{d}(M)}\left(W_{\mathfrak{s l}_{2}}\left(Z^{\mathrm{LMO}}(N)\right) \cdot \exp \left(W_{\mathfrak{s l}_{2}}(\text { the constant terms })\right)\right)^{d},
$$

and

$$
\tau\left(M_{d} ; 0\right) \sim “(32) ” \cdot\left(-\frac{1}{2}\right) \operatorname{Res}_{t=0}\left(1-t^{-1}\right)^{2}=“(32) ” .
$$

Therefore, the degree $\leq \ell$ part of $\left(q^{-(3 / 4) \sigma_{d}(M)} \tau\left(M_{d} ; 0\right)\right)^{1 / d}$ is stable for sufficiently large $d$.

Proposition 5.6 Suppose that $\Delta(t)$ has no multiple zeros, and let $c_{1}^{ \pm 1}, \ldots, c_{n}^{ \pm 1}$ be the zeros of $\Delta(t)$. Let $c$ be 0 or a zero of $\Delta(t)$. Let $d$ be a positive integer satisfying that $\Delta(t)$ does not have a zero of a $d$ th root of unity (that is, $b_{1}\left(M_{d}\right)=1$ ). Then, 
(1) $\lambda_{0}\left(M_{d} ; c^{d}\right)$ is presented by a rational function of $c_{1}^{d}, \ldots, c_{n}^{d}$.

(2) $\left(\lambda_{1}\left(M_{d} ; c^{d}\right)-\frac{3}{4} \sigma_{d}(M) \lambda_{0}\left(M_{d} ; c^{d}\right)\right) / d$ is stable, in the sense that it can be presented by a rational function of $c_{1}^{d}, \ldots, c_{n}^{d}$ for sufficiently large $d$ satisfying the above condition.

Hence, the stable part can be presented by

$$
\tilde{\lambda}_{1}(M ; c)=\left(\left(\lambda_{1}\left(M_{d} ; c^{d}\right)-\frac{3}{4} \sigma_{d}(M) \lambda_{0}\left(M_{d} ; c^{d}\right)\right) / d \text { for such } d \gg 0\right),
$$

where this is a rational function of $c_{1}^{d}, \ldots, c_{n}^{d}$.

Proof of Proposition 5.6 We show (1), as follows. We put

$$
\Delta(t)=b \prod_{i}\left(1-a_{i}\left(t+t^{-1}-2\right)\right),
$$

where $a_{i}^{-1}=c_{i}+c_{i}^{-1}-2$. Then

$$
\Delta_{K_{d}}(t) / \Delta_{K_{d}}(1)=\prod_{i}\left(1-\tilde{a}_{i}\left(t+t^{-1}-2\right)\right),
$$

where ${\tilde{a_{i}}}^{-1}=c_{i}^{d}+c_{i}^{-d}-2$. Hence, by Proposition 5.3, we obtain (1).

We show (2), in the following of this proof. Similarly as the proof of Proposition 5.5, $\chi^{-1} Z^{\mathrm{LMO}}\left(N_{d}, K_{d}\right) \sim$

$$
\begin{gathered}
e^{\sigma_{d}(M) \theta / 16} \sqcup Z^{\mathrm{LMO}}(N)^{d} \sqcup \Omega \sqcup \exp _{\sqcup}(\overbrace{}^{-\frac{1}{2} \log \left(\Delta_{K_{d}}(t) / \Delta_{K_{d}}(1)\right)}) \\
=e^{\sigma_{d}(M) \theta / 16} \sqcup \Omega \sqcup \exp _{\sqcup}\left(C^{\left.-\frac{1}{2} \log \left(\Delta_{K_{d}}(t) / \Delta_{K_{d}}(1)\right)\right)}\right) \\
\sqcup(1+(2-\text { loop part })+\cdots),
\end{gathered}
$$

where the 2-loop part is determined by the 2-loop polynomial $\Theta_{K_{d}}\left(t_{1}, t_{2}\right)$ (see Garoufalidis-Kricker [8] and the author's paper [33]),

$$
\begin{aligned}
\frac{\Theta_{K_{d}}\left(t_{1}, t_{2}\right)}{\Delta_{K_{d}}\left(t_{1}\right) \Delta_{K_{d}}\left(t_{2}\right) \Delta_{K_{d}}\left(t_{1} t_{2}\right)} & = \\
& \frac{1}{d} \sum_{\zeta_{1}^{d}=\zeta_{2}^{d}=1} \frac{\Theta\left(\zeta_{1} t_{1}^{1 / d}, \zeta_{2} t_{2}^{1 / d}\right)}{\Delta\left(\zeta_{1} t_{1}^{1 / d}\right) \Delta\left(\zeta_{2} t_{2}^{1 / d}\right) \Delta\left(\zeta_{1} \zeta_{2} t_{1}^{1 / d} t_{2}^{1 / d}\right)},
\end{aligned}
$$


where $\Theta\left(t_{1}, t_{2}\right)$ denotes the 2-loop polynomial of $K$. Hence, the $\mathfrak{s l}_{2}$ reduction of $\chi^{-1} Z^{\mathrm{LMO}}\left(N_{d}, K_{d}\right) \sqcup \Omega^{-1}$ is stably presented by

$$
q^{(3 / 4) \sigma_{d}(M)}\left(\frac{\Delta_{K_{d}}(1)}{\Delta_{K_{d}}(t)}+\frac{\Theta_{K_{d}}(t, 1)}{\Delta_{K_{d}}(t)^{3}}(q-1)+\cdots\right) .
$$

Further,

$$
\begin{aligned}
& \lambda_{0}\left(M_{d} ; c^{d}\right)=-\frac{1}{2} \cdot \frac{1+c^{d}}{1-c^{d}} \operatorname{Res}_{t=c} \frac{\left(1-t^{-1}\right)^{2} \Delta_{K_{d}}(1)}{\Delta_{K_{d}}(t)}, \\
& \lambda_{1}\left(M_{d} ; c^{d}\right)=\frac{3}{4} \sigma_{d}(M) \lambda_{0}\left(M_{d} ; c^{d}\right)-\frac{1}{2} \cdot \frac{1+c^{d}}{1-c^{d}} \operatorname{Res}_{t=c} \frac{\left(1-t^{-1}\right)^{2} \Theta_{K_{d}}(t, 1)}{\Delta_{K_{d}}(t)^{3}} .
\end{aligned}
$$

Therefore, it is sufficient to show that $\Theta_{K_{d}}(t, 1) / d$ is presented by a rational function of $c_{1}^{d}, \ldots, c_{n}^{d}$ for $d \gg 0$.

We show it, in the following of this proof. For example, when $\Delta(t)=1-a\left(t+t^{-1}-2\right)$,

$$
\Delta_{K_{d}}(t)=a^{d}\left(c^{d}+c^{-d}-t-t^{-1}\right)
$$

for a zero $c$ of $\Delta(t)$. Further, since

$$
\begin{aligned}
\frac{\Delta_{K_{d}}\left(s^{d}\right)}{\Delta(s)} & =a^{d-1} s^{-d+1} \frac{\left(s^{d}-c^{d}\right)\left(s^{d}-c^{-d}\right)}{(s-c)\left(s-c^{-1}\right)} \\
& =\frac{a^{d-1} s^{-d+1}}{c-c^{-1}}\left(\frac{s^{d}-c^{d}}{s-c}\left(s^{d}-c^{-d}\right)-\frac{s^{d}-c^{-d}}{s-c^{-1}}\left(s^{d}-c^{d}\right)\right),
\end{aligned}
$$

we can calculate $\Theta_{K_{d}}\left(t_{1}, t_{2}\right)$ by (33) putting $s=\zeta_{i} t_{i}^{1 / d}$. For example, when $\Theta\left(t_{1}, t_{2}\right)=$ 1 , the summand of (33) is presented by

$$
\frac{d a^{3 d-3}}{\left(c-c^{-1}\right)^{3}} \prod_{i=1}^{3}\left(\frac{s_{i}^{d}-c^{d}}{s_{i}-c}\left(s_{i}^{d}-c^{-d}\right)-\frac{s_{i}^{d}-c^{-d}}{s_{i}-c^{-1}}\left(s_{i}^{d}-c^{d}\right)\right),
$$

where we put $s_{i}=\zeta_{i} t_{1}^{1 / d}$, and $s_{1} s_{2} s_{3}=1$. Since the sum of (33) picks up the terms of the form $s_{1}^{n_{1} d} s_{2}^{n_{2} d}$ for $n_{1}, n_{2} \in \mathbb{Z}$, we pick up the following term, for example, from a part of the above product,

$$
\begin{aligned}
\frac{d a^{3 d-3}}{\left(c-c^{-1}\right)^{3}} \cdot \frac{s_{1}^{d}-c^{d}}{s_{1}-c} \frac{s_{2}^{d}-c^{d}}{s_{2}-c} \frac{s_{3}^{d}-c^{d}}{s_{3}-c} \cdot\left(s_{1}^{d}-c^{-d}\right)\left(s_{2}^{d}-c^{-d}\right)\left(s_{3}^{d}-c^{-d}\right) \rightsquigarrow & \rightsquigarrow \\
& \frac{d a^{3 d-3}}{\left(c-c^{-1}\right)^{3}} \cdot \frac{c^{3 d}-1}{c^{3}-1} \cdot\left(t_{1}-c^{-d}\right)\left(t_{2}-c^{-d}\right)\left(t_{3}-c^{-d}\right) .
\end{aligned}
$$


Hence, in this way, we can show that

$$
\begin{aligned}
\frac{\Theta_{K_{d}}\left(t_{1}, t_{2}\right)}{d}= & \frac{a^{3 d-3}(c+1)\left(c^{d}-1\right)^{2}}{\left(c^{2}-1\right)^{3}\left(c^{3}-1\right) c^{d-4}} \\
& \times\left(\frac{\left(c^{d-1}-1\right)\left(c^{d+1}-1\right)}{c^{d}}\left(t_{1} t_{2}+t_{1}+t_{2}+t_{1}^{-1}+t_{2}^{-1}+t_{1}^{-1} t_{2}^{-1}\right)\right. \\
& \left.\quad+\frac{\left(c^{d}-1\right)^{2}}{c^{d}}\left(\left(c^{d}+c^{-d}\right)\left(c+c^{-1}-1\right)+4 c+4 c^{-1}+2\right)\right) .
\end{aligned}
$$

For general $\Theta\left(t_{1}, t_{2}\right)$ and $\Delta(t)$, we can similarly show that $\Theta_{K_{d}}(t, 1) / d$ is presented by a rational function of $c_{1}^{d}, \ldots, c_{n}^{d}$ for $d \gg 0$.

\section{Arithmetic limits of rational functions of roots of unity}

The aim of this section is to show Proposition 6.1, which is used when we calculate arithmetic expansion of quantum invariants in Section 1.

Let $p$ be an odd prime. We put $\zeta=\exp (2 \pi \sqrt{-1} / p)$. We review some preliminaries. It is known that $p$ is divisible by $(\zeta-1)$ precisely $p-1$ times in $\mathbb{Z}[\zeta]$ (because $p=$ $(-1)^{(p-1) / 2} \prod_{i=1}^{(p-1) / 2}\left(\zeta^{2 i-1}-\zeta^{-(2 i-1)}\right)^{2}$; see, for example, Ireland and Rosen [13, Proposition 6.4.2]). For non-negative integer $i$ and an indeterminate $x$, the binomial coefficient $\left(\begin{array}{l}x \\ i\end{array}\right)$ is defined by

$$
\left(\begin{array}{l}
x \\
i
\end{array}\right)=\frac{1}{i !} x(x-1)(x-2) \ldots(x-i+1)
$$

Proposition 6.1 We set $\varphi(t)$ by $\varphi(t)=f(t) / g(t)$ where $f(t), g(t) \in \mathbb{Z}_{(p)}\left[t, t^{-1}\right]$ with $f(t)=f\left(t^{-1}\right), g(t)=g\left(t^{-1}\right)$ and $g(1)$ is not divisible by $p$. Then,

$$
\frac{1}{p} \sum_{n \in \mathbb{Z} / p \mathbb{Z}} \varphi\left(\zeta^{n}\right) \in \mathbb{Z}_{(p)}[\zeta] .
$$

Further, if $p>\operatorname{deg} f(t)-\operatorname{deg} g(t)$, then,

$$
\frac{1}{p} \sum_{n \in \mathbb{Z} / p \mathbb{Z}} \varphi\left(\zeta^{n}\right)=\sum_{c=0, c_{1}, \ldots, c_{n}}\left(\frac{1+c}{1-c}\right)^{p} \operatorname{Res}_{t=c} \frac{\varphi(t)}{t}+O\left((\zeta-1)^{p-1} ; \mathbb{Z}_{(p)}[\zeta]\right),
$$

where $c_{1}, c_{1}^{-1}, \ldots, c_{n}, c_{n}^{-1}$ are the zeros of $g(t)$.

To prove Proposition 6.1, we show some lemmas, which give particular cases of Proposition 6.1. 
Lemma 6.2 Let $\varphi(t)$ be a polynomial in $\mathbb{Q}\left[t, t^{-1}\right]$ with $\varphi(t)=\varphi\left(t^{-1}\right)$. If $p>$ $\operatorname{deg} \varphi(t)$, then

$$
\frac{1}{p} \sum_{n \in \mathbb{Z} / p \mathbb{Z}} \varphi\left(\zeta^{n}\right)=\operatorname{Res}_{t=0} \frac{\varphi(t)}{t}
$$

Proof It is sufficient to show the lemma when $\varphi(t)=t^{i}$ for $|i|<p$. Then, both sides of the required formula are equal to 1 if $i=0,0$ otherwise.

Lemma 6.3 We set $\varphi(t)$ by $\varphi(t)=f(t) / g(t)$ where $g(t)=1-b\left(t+t^{-1}-2\right)$ with $b \in \mathbb{Z}_{(p)}$ and $f(t) \in \mathbb{Z}_{(p)}\left[t, t^{-1}\right]$ with $f(t)=f\left(t^{-1}\right)$. If $p>\operatorname{deg} f(t)-\operatorname{deg} g(t)$, then

$$
\frac{1}{p} \sum_{n \in \mathbb{Z} / p \mathbb{Z}} \varphi\left(\zeta^{n}\right)=\sum_{c=0, c_{1}}\left(\frac{1+c}{1-c}\right)^{p} \operatorname{Res}_{t=c} \frac{\varphi(t)}{t}+O\left((\zeta-1)^{p-1} ; \mathbb{Z}_{(p)}[\zeta]\right),
$$

where $c_{1}$ is a zero of $g(t)$.

Proof We assume that $b$ is not divisible by $p$ (otherwise, the lemma is reduced to Lemma 6.2). Further, by Lemma 6.2, we can reduce the lemma by subtracting a polynomial from $\varphi(t)$. Hence, it is sufficient to show the lemma when $f(t)=1$. Putting $\eta=\zeta^{n}+\zeta^{-n}-2$, we expand

$$
\frac{1}{1-b \eta}=\sum_{0 \leq i<p} b^{i} \eta^{i}+\frac{\eta^{p}}{1-b \eta} \equiv \sum_{0 \leq i<p} b^{i} \eta^{i},
$$

since $\eta^{p} \in O\left((\zeta-1)^{2 p}\right)$ and $1-b \eta$ is invertible in $\mathbb{Z}_{(p)}[\zeta]$, where the equivalence in the formula means the equivalence modulo $O\left((\zeta-1)^{2 p}\right)$. Further, for $i<p$,

$$
\begin{aligned}
\frac{1}{p} \sum_{n \in \mathbb{Z} / p \mathbb{Z}} \eta^{i} & =\frac{1}{p} \sum_{n \in \mathbb{Z} / p \mathbb{Z}}\left(\zeta^{n}+\zeta^{-n}-2\right)^{i} \\
& =\left(\text { constant term of }\left(t+t^{-1}-2\right)^{i}=\left(t^{1 / 2}-t^{-1 / 2}\right)^{2 i}\right) \\
& =(-1)^{i}\left(\begin{array}{c}
2 i \\
i
\end{array}\right)=4^{i}\left(\begin{array}{c}
-1 / 2 \\
i
\end{array}\right) .
\end{aligned}
$$

Hence

$$
\begin{aligned}
\frac{1}{p} \sum_{n \in \mathbb{Z} / p \mathbb{Z}} \frac{1}{1-b \eta} & \equiv \sum_{0 \leq i<p} b^{i} 4^{i}\left(\begin{array}{c}
-1 / 2 \\
i
\end{array}\right) \underset{\equiv(p)}{\equiv} \sum_{i=0}^{(p-1) / 2}(4 b)^{i}\left(\begin{array}{c}
(p-1) / 2 \\
i
\end{array}\right) \\
& \equiv(4 b+1)^{(p-1) / 2}=\left(\frac{1+c_{1}}{1-c_{1}}\right)^{p-1},
\end{aligned}
$$


since $b^{-1}=c_{1}+c_{1}^{-1}-2$. Further,

$$
\begin{aligned}
& \operatorname{Res}_{t=0} \frac{1}{\operatorname{tg}(t)}=0, \\
& \operatorname{Res}_{t=c_{1}} \frac{1}{\operatorname{tg}(t)}=\lim _{t \rightarrow c_{1}} \frac{t-c_{1}}{\operatorname{tg}(t)}=\frac{1}{c_{1} g^{\prime}\left(c_{1}\right)}=-\frac{1}{b\left(c_{1}-c_{1}^{-1}\right)}=\frac{1-c_{1}}{1+c_{1}} .
\end{aligned}
$$

Therefore, noting that $p \in O\left((\zeta-1)^{p-1}\right)$, we obtain the required formula.

Lemma 6.4 For a positive integer $\ell$, we set $\varphi(t)$ by $\varphi(t)=f(t) / g(t)^{\ell}$, where $f(t)$ and $g(t)$ are as in Lemma 6.3. If $p>\operatorname{deg} f(t)-\operatorname{deg} g(t)^{\ell}$, then

$$
\frac{1}{p} \sum_{n \in \mathbb{Z} / p \mathbb{Z}} \varphi\left(\zeta^{n}\right)=\sum_{c=0, c_{1}}\left(\frac{1+c}{1-c}\right)^{p} \operatorname{Res}_{t=c} \frac{\varphi(t)}{t}+O\left((\zeta-1)^{p-1} ; \mathbb{Z}_{(p)}[\zeta]\right),
$$

where $c_{1}$ is a zero of $g(t)$.

Proof It is sufficient to show the lemma for $\varphi(t)=\left(t+t^{-1}-2\right)^{\ell-1} /\left(1-b\left(t+t^{-1}-2\right)\right)^{\ell}$, because other $\varphi(t)$ can be obtained as a linear sum of a polynomial and such $\varphi(t)$. Similarly as (34), putting $\eta=\zeta^{n}+\zeta^{-n}-2$, we have that

$$
\begin{aligned}
\frac{1}{p} \sum_{n \in \mathbb{Z} / p \mathbb{Z}} \frac{\eta^{\ell-1}}{(1-b \eta)^{\ell}} & \equiv \sum_{0 \leq i<p-\ell+1}(-1)^{i}\left(\begin{array}{c}
-\ell \\
i
\end{array}\right) b^{i} \eta^{i+\ell-1} \\
& =\sum_{0 \leq i<p-\ell+1}(-1)^{i}\left(\begin{array}{c}
-\ell \\
i
\end{array}\right) b^{i} 4^{i+\ell-1}\left(\begin{array}{c}
-1 / 2 \\
i+\ell-1
\end{array}\right) .
\end{aligned}
$$

Further,

$$
\begin{aligned}
(-1)^{i}\left(\begin{array}{c}
-\ell \\
i
\end{array}\right) 4^{\ell-1}\left(\begin{array}{c}
-1 / 2 \\
i+\ell-1
\end{array}\right) & =4^{\ell-1}\left(\begin{array}{c}
i+\ell-1 \\
i
\end{array}\right)\left(\begin{array}{c}
-1 / 2 \\
i+\ell-1
\end{array}\right) \\
& =4^{\ell-1}\left(\begin{array}{c}
-1 / 2 \\
\ell-1
\end{array}\right)\left(\begin{array}{c}
1 / 2-\ell \\
i
\end{array}\right),
\end{aligned}
$$

and

$$
\begin{aligned}
4^{\ell-1}\left(\begin{array}{c}
-1 / 2 \\
\ell-1
\end{array}\right) & =\frac{4^{\ell-1}}{(\ell-1) !}\left(-\frac{1}{2}\right)\left(-\frac{3}{2}\right) \cdots\left(-\frac{2 \ell-3}{2}\right) \\
& =\frac{2^{\ell-1}}{(\ell-1) !}(-1)^{\ell+1}(2 \ell-3) ! !=(-1)^{\ell+1} 2\left(\begin{array}{c}
2 \ell-3 \\
\ell-1
\end{array}\right) .
\end{aligned}
$$


Hence,

$$
\begin{aligned}
\frac{1}{p} \sum_{n \in \mathbb{Z} / p \mathbb{Z}} \frac{\eta^{\ell-1}}{(1-b \eta)^{\ell}} & \equiv(-1)^{\ell+1} 2\left(\begin{array}{c}
2 \ell-3 \\
\ell-1
\end{array}\right) \sum_{0 \leq i \leq(p+1) / 2-\ell}\left(\begin{array}{c}
1 / 2-\ell \\
i
\end{array}\right)(4 b)^{i} \\
& \equiv(-1)^{\ell+1} 2\left(\begin{array}{c}
2 \ell-3 \\
\ell-1
\end{array}\right)(4 b+1)^{(p+1) / 2-\ell} \\
& =(-1)^{\ell+1} 2\left(\begin{array}{c}
2 \ell-3 \\
\ell-1
\end{array}\right)\left(\frac{1+c_{1}}{1-c_{1}}\right)^{p-2 \ell+1} .
\end{aligned}
$$

Further,

$$
\begin{aligned}
& \operatorname{Res}_{t=0} \frac{\left(t+t^{-1}-2\right)^{\ell-1}}{t\left(1-b\left(t+t^{-1}-2\right)\right)^{\ell}}=0, \\
& \operatorname{Res}_{t=c_{1}} \frac{\left(t+t^{-1}-2\right)^{\ell-1}}{t\left(1-b\left(t+t^{-1}-2\right)\right)^{\ell}}=(-1)^{\ell+1} 2\left(\begin{array}{c}
2 \ell-3 \\
\ell-1
\end{array}\right)\left(\frac{1-c_{1}}{1+c_{1}}\right)^{2 \ell-1},
\end{aligned}
$$

where the second formula is obtained by Lemma 6.5 below. Hence, we obtain the required formula for $\varphi(t)=\left(t+t^{-1}-2\right)^{\ell-1} /\left(1-b\left(t+t^{-1}-2\right)\right)^{\ell}$.

Lemma 6.5 Put $z=t+t^{-1}-2$, and let $t=c$ be a zero of $1-b z$. Then

(1) $\operatorname{Res}_{t=c} \frac{z^{\ell}}{t(1-b z)^{\ell+1}}=(-1)^{\ell} 2\left(\begin{array}{c}2 \ell-1 \\ \ell\end{array}\right)\left(\frac{1-c}{1+c}\right)^{2 \ell+1}$,

(2) $\ell \operatorname{Res}_{t=c} \frac{(\ell-2 b) z^{\ell}-b z^{\ell+1}}{t(1-b z)^{\ell}}=(-1)^{\ell} 2 \ell\left(\begin{array}{c}2 \ell-1 \\ \ell\end{array}\right)\left(\frac{1-c}{1+c}\right)^{2 \ell-1}$.

Proof We show that " $(1)_{\ell} \Leftrightarrow(2)_{\ell}$ ", as follows. The functions of the residues of $(1)_{\ell}$ and $(2)_{\ell}$ are related by

$$
\frac{z^{\ell}}{t(1-b z)^{\ell+1}}-\frac{b}{\ell(4 b+1)} \cdot \frac{d}{d t}\left(\frac{\left(t-t^{-1}\right) z^{\ell}}{(1-b z)^{\ell}}\right)=\frac{1}{\ell(4 b+1)} \cdot \frac{(\ell-2 b) z^{\ell}-b z^{\ell+1}}{t(1-b z)^{\ell}} .
$$

Since the differential of a function does not contribute to the residue, $(1)_{\ell}$ is equivalent to $(2)_{\ell}$, noting that $4 b+1=(1+c)^{2} /(1-c)^{2}$.

We show that " $(2)_{\ell}+b(2)_{\ell+1}=(\ell-2 b)(1)_{\ell}$ ", as follows. We have that $\left(\right.$ LHS of $\left.(2)_{\ell}\right)+b\left(\right.$ LHS of $\left.(2)_{\ell+1}\right)$

$$
\begin{aligned}
=\operatorname{Res}_{t=c} \frac{(1-b z)\left((\ell-2 b) z^{\ell}-b z^{\ell+1}\right)+b\left((\ell+1-2 b) z^{\ell+1}-b z^{\ell+2}\right)}{t(1-b z)^{\ell+1}} \\
=\operatorname{Res}_{t=c} \frac{(\ell-2 b) z^{\ell}}{t(1-b z)^{\ell+1}}=(\ell-2 b)\left(\text { LHS of }(1)_{\ell}\right) .
\end{aligned}
$$


Further, it is shown by elementary calculation that

$$
\left(\text { RHS of }(2)_{\ell}\right)+b\left(\text { RHS of }(2)_{\ell+1}\right)=(\ell-2 b)\left(\text { RHS of }(1)_{\ell}\right) \text {. }
$$

Hence, if (1) $\ell$ and $(2)_{\ell}$ hold, then $(2)_{\ell+1}$ holds.

Therefore, by induction, $(1)_{\ell}$ and $(2)_{\ell}$ hold for all $\ell$.

Proof of Proposition 6.1 We can assume that $g(1)=1$ by replacing $g(t)$ with its quotient by $g(1)$.

If $g(t)$ is of degree 2 , we put $g(t)=\left(1-b_{1}\left(t+t^{-1}-2\right)\right)\left(1-b_{2}\left(t+t^{-1}-2\right)\right)$ where $b_{1}$ and $b_{2}$ are complex number in general, but $b_{1}+b_{2}, b_{1} b_{2} \in \mathbb{Z}_{(p)}$. If $\left(b_{1}-b_{2}\right)^{2}$ is divisible by $p$, the proposition is reduced to Lemma 6.4. We assume that $\left(b_{1}-b_{2}\right)^{2}$ is not divisible by $p$. We can put $f(t)=f_{0}+f_{1}\left(t+t^{-1}-2\right)$ by subtracting a polynomial from $\varphi(t)$. Then,

$$
\varphi(t)=\frac{f(t)}{g(t)}=\frac{1}{b_{1}-b_{2}}\left(\frac{b_{1} f_{0}+f_{1}}{1-b_{1}\left(t+t^{-1}-2\right)}-\frac{b_{2} f_{0}+f_{1}}{1-b_{2}\left(t+t^{-1}-2\right)}\right) .
$$

Further, in the same way as the proof of (34),

$$
\frac{1}{p} \sum_{n \in \mathbb{Z} / p \mathbb{Z}} \varphi\left(\zeta^{n}\right) \equiv \frac{1}{b_{1}-b_{2}}\left(\left(b_{1} f_{0}+f_{1}\right)\left(\frac{1+c_{1}}{1-c_{1}}\right)^{p-1}-\left(b_{2} f_{0}+f_{1}\right)\left(\frac{1+c_{2}}{1-c_{2}}\right)^{p-1}\right),
$$

where $c_{i}$ is a zero of $1-b_{i}\left(t+t^{-1}-2\right)$. Since

$$
\begin{aligned}
& \operatorname{Res}_{t=0} \frac{\varphi(t)}{t}=0, \\
& \operatorname{Res}_{t=c_{i}} \frac{\varphi(t)}{t}=\lim _{t \rightarrow c_{i}} \frac{\left(t-c_{i}\right) f(t)}{\operatorname{tg}(t)}=\frac{f\left(c_{i}\right)}{c_{i} g^{\prime}\left(c_{i}\right)}=\frac{b_{i} f_{0}+f_{1}}{b_{i}-b_{3-i}} \cdot \frac{1-c_{i}}{1+c_{i}},
\end{aligned}
$$

we obtain the required formula in this case.

For a general $g(t)$, we can present $\varphi(t)$ as a linear sum of the $\varphi(t)$ of Lemmas 6.3 and 6.4. Hence, we can show the required formula in the same way as above.

\section{References}

[1] D Bar-Natan, S Garoufalidis, L Rozansky, D P Thurston, The Århus integral of rational homology 3-spheres I: A highly non trivial flat connection on $S^{3}$, Selecta Math. (N.S.) 8 (2002) 315-339 MR1931167

[2] D Bar-Natan, S Garoufalidis, L Rozansky, DP Thurston, The Århus integral of rational homology 3-spheres II: Invariance and universality, Selecta Math. (N.S.) 8 (2002) 341-371 MR1931168 
[3] D Bar-Natan, S Garoufalidis, L Rozansky, D P Thurston, The Arhus integral of rational homology 3-spheres III: Relation with the Le-Murakami-Ohtsuki invariant, Selecta Math. (N.S.) 10 (2004) 305-324 MR2099069

[4] S V Chmutov, A N Varchenko, Remarks on the Vassiliev knot invariants coming from $\mathrm{sl}_{2}$, Topology 36 (1997) 153-178 MR1410469

[5] R Fenn, C Rourke, On Kirby's calculus of links, Topology 18 (1979) 1-15 MR528232

[6] S Garoufalidis, N Habegger, The Alexander polynomial and finite type 3-manifold invariants, Math. Ann. 316 (2000) 485-497 MR1752781

[7] S Garoufalidis, A Kricker, A surgery view of boundary links, Math. Ann. 327 (2003) 103-115 MR2005123

[8] S Garoufalidis, A Kricker, Finite type invariants of cyclic branched covers, Topology 43 (2004) 1247-1283 MR2081426

[9] S Garoufalidis, A Kricker, A rational noncommutative invariant of boundary links, Geom. Topol. 8 (2004) 115-204 MR2033481

[10] N Habegger, A computation of the universal quantum 3-manifold invariant for manifolds of rank greater than 2, preprint (1996) Available at http:// www.math.sciences. univ-nantes.fr/ habegger/

[11] N Habegger, A Beliakova, The Casson-Walker-Lescop invariant as a quantum 3manifold invariant, J. Knot Theory Ramifications 9 (2000) 459-470 MR1758865

[12] K Habiro, Claspers and finite type invariants of links, Geom. Topol. 4 (2000) 1-83 MR1735632

[13] K Ireland, M Rosen, A classical introduction to modern number theory, second edition, Graduate Texts in Mathematics 84, Springer, New York (1990) MR1070716

[14] R Kirby, P Melvin, The 3-manifold invariants of Witten and Reshetikhin-Turaev for sl(2, C), Invent. Math. 105 (1991) 473-545 MR1117149

[15] S Kojima, M Yamasaki, Some new invariants of links, Invent. Math. 54 (1979) 213228 MR553219

[16] M Kontsevich, Vassiliev's knot invariants, from: “I. M. Gel'fand Seminar", Adv. Soviet Math. 16, Amer. Math. Soc., Providence, RI (1993) 137-150 MR1237836

[17] A Kricker, The lines of the Kontsevich integral and Rozansky's rationality conjecture arXiv:math.GT/0005284

[18] A Kricker, A surgery formula for the 2-loop piece of the LMO invariant of a pair, from: "Invariants of knots and 3-manifolds (Kyoto, 2001)", Geom. Topol. Monogr. 4, Geom. Topol. Publ., Coventry (2002) 161-181 MR2002609

[19] T T Q Le, J Murakami, T Ohtsuki, On a universal perturbative invariant of 3manifolds, Topology 37 (1998) 539-574 MR1604883 
[20] C Lescop, Sur l'invariant de Casson-Walker: formule de chirurgie globale et généralisation aux variétés de dimension 3 fermées orientées, C. R. Acad. Sci. Paris Sér. I Math. 315 (1992) 437-440 MR1179053

[21] C Lescop, Global surgery formula for the Casson-Walker invariant, Annals of Mathematics Studies 140, Princeton University Press, Princeton, NJ (1996) MR1372947

[22] J Lieberum, The LMO-invariant of 3-manifolds of rank one and the Alexander polynomial, Math. Ann. 318 (2000) 761-776 MR1802509

[23] H Murakami, Quantum SO(3)-invariants dominate the SU(2)-invariant of Casson and Walker, Math. Proc. Cambridge Philos. Soc. 117 (1995) 237-249 MR1307078

[24] J Neukirch, Algebraic number theory, Grundlehren der Mathematischen Wissenschaften 322, Springer, Berlin (1999) MR1697859 Translated from the 1992 German original and with a note by Norbert Schappacher. With a foreword by G Harder

[25] T Ohtsuki, A refinement of the LMO invariant for 3-manifolds with the first Betti number 1 , in preparation

[26] T Ohtsuki, A polynomial invariant of rational homology 3-spheres, Invent. Math. 123 (1996) 241-257 MR1374199

[27] T Ohtsuki, The perturbative $\mathrm{SO}(3)$ invariant of rational homology 3-spheres recovers from the universal perturbative invariant, Topology 39 (2000) 1103-1135 MR1783850

[28] T Ohtsuki, Quantum invariants, Series on Knots and Everything 29, World Scientific Publishing Co., River Edge, NJ (2002) MR1881401

[29] T Ohtsuki, Invariants of knots and 3-dimensional manifolds, Sūgaku 55 (2003) 337349 MR2021547

[30] T Ohtsuki, A cabling formula for the 2-loop polynomial of knots, Publ. Res. Inst. Math. Sci. 40 (2004) 949-971 MR2074706

[31] T Ohtsuki, Invariants of knots derived from equivariant linking matrices of their surgery presentations, preprint (2006)

[32] T Ohtsuki, Equivariant quantum invariants of the infinite cyclic covers of knot complements, from: "Intelligence of low dimensional topology 2006", Ser. Knots Everything 40, World Sci. Publ., Hackensack, NJ (2007) 253-262 MR2371733

[33] T Ohtsuki, On the 2-loop polynomial of knots, Geom. Topol. 11 (2007) 1357-1475 MR2326948

[34] N Reshetikhin, V G Turaev, Invariants of 3-manifolds via link polynomials and quantum groups, Invent. Math. 103 (1991) 547-597 MR1091619

[35] P Ribenboim, Classical theory of algebraic numbers, Universitext, Springer, New York (2001) MR1821363

[36] L Rozansky, A contribution of a U(1)-reducible connection to quantum invariants of links I: $R$-matrix and Burau representation arXiv:math/9806004 
[37] L Rozansky, A contribution of a U(1)-reducible connection to quantum invariants of links II: Links in rational homology spheres arXiv:math/9806066

[38] L Rozansky, Witten's invariants of rational homology spheres at prime values of $K$ and trivial connection contribution, Comm. Math. Phys. 180 (1996) 297-324 MR1405953

[39] L Rozansky, Higher order terms in the Melvin-Morton expansion of the colored Jones polynomial, Comm. Math. Phys. 183 (1997) 291-306 MR1461960

[40] L Rozansky, On p-adic convergence of perturbative invariants of some rational homology spheres, Duke Math. J. 91 (1998) 353-379 MR1600594

[41] L Rozansky, The universal $R$-matrix, Burau representation, and the Melvin-Morton expansion of the colored Jones polynomial, Adv. Math. 134 (1998) 1-31 MR1612375

[42] L Rozansky, A rational structure of generating functions for Vassiliev invariants, notes accompanying lectures at the Summer School on Quantum Invariants of Knots and Three-Manifolds, Joseph Fourier Institute, University of Grenoble (1999)

[43] K Walker, An extension of Casson's invariant, Annals of Mathematics Studies 126, Princeton University Press, Princeton, NJ (1992) MR1154798

[44] E Witten, Quantum field theory and the Jones polynomial, Comm. Math. Phys. 121 (1989) 351-399 MR990772

Research Institute for Mathematical Sciences, Kyoto University

Sakyo-ku, Kyoto, 606-8502, Japan

tomotada@kurims.kyoto-u.ac.jp

http://www.kurims.kyoto-u.ac.jp/ tomotada/

Proposed: Vaughan Jones

Seconded: Joan Birman, Shigeyuki Morita.

Received: 28 August 2009

Revised: 27 May 2010 